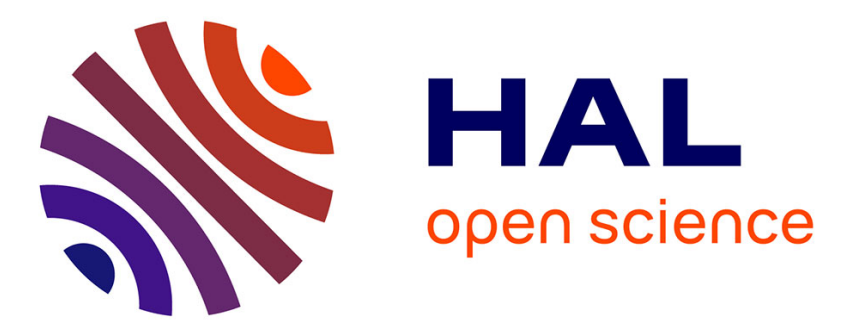

\title{
Estimations of the effective conductivity of anisotropic multiphase composites with imperfect interfaces
}

\author{
Hung Le Quang, D. C. Pham, Guy Bonnet, Qi-Chang He
}

\section{To cite this version:}

Hung Le Quang, D. C. Pham, Guy Bonnet, Qi-Chang He. Estimations of the effective conductivity of anisotropic multiphase composites with imperfect interfaces. International Journal of Heat and Mass Transfer, 2013, 58 (1-2), pp.175-187. hal-00771215

\section{HAL Id: hal-00771215 \\ https://hal.science/hal-00771215}

Submitted on 6 Mar 2016

HAL is a multi-disciplinary open access archive for the deposit and dissemination of scientific research documents, whether they are published or not. The documents may come from teaching and research institutions in France or abroad, or from public or private research centers.
L'archive ouverte pluridisciplinaire HAL, est destinée au dépôt et à la diffusion de documents scientifiques de niveau recherche, publiés ou non, émanant des établissements d'enseignement et de recherche français ou étrangers, des laboratoires publics ou privés. 


\title{
Estimations of the effective conductivity of anisotropic multiphase composites with imperfect interfaces
}

\author{
Hung Le Quang ${ }^{\mathrm{a}, *}$, Duc Chinh Pham ${ }^{\mathrm{b}}$, Guy Bonnet ${ }^{\mathrm{a}}$, Qi-Chang He ${ }^{\mathrm{a}}$ \\ a Université Paris-Est, Laboratoire de Modélisation et Simulation Multi Echelle, UMR 8208 CNRS, 5 Bd. Descartes, 77454 Marne-la-Vallée Cedex 2, France \\ b Institute of Mechanics - VAST, 264 Doi Can, Hanoi, Viet Nam
}

\section{A B S T R A C T}

In this work, approximation schemes are developed to estimate the effective conductivity or resistivity of composites made of several phases with imperfect interfaces. The interface can be either highly conducting or resistive, and the constituent phases can be anisotropic. By using a generalized Eshelby's tensor accounting for imperfect interfaces and by applying the dilute distribution, Mori-Tanaka, self-consistent and generalized self-consistent schemes while incorporating imperfect interfaces between the inhomogeneity and matrix phases, the closed-form expressions for the effective conductivity and resistivity tensors are obtained. With the help of the dilute solution results for an inhomogeneity embedded in an effective medium matrix via an imperfect interface, the differential scheme is extended to predicting the effective conductivity and resistivity tensors. The estimations obtained by the differential scheme for the effective conductivity and resistivity are shown to comply with the generalized Hashin-Shtrikman bounds. Numerical results are provided to illustrate the dependence of the effective conductivity on the size and orientation distribution of inhomogeneities and to compare the estimations with the relevant upper and lower bounds.

Keywords:

Interface

Highly conducting interface

Kapitza interface thermal resistance

Anisotropy

Thermal conduction

Composite materials

\section{Introduction}

In most of the classical approximation schemes dedicated to estimating the effective properties of inhomogeneous materials, it is often assumed that the interfaces between the constituent phases are perfect. In the context of thermal conduction phenomenon, an interface is called perfect if both temperature and normal heat flux are continuous across it. However, in practice, due to the presence of roughness or mismatch between the phases, for example, the assumption of perfect interface is not appropriate and imperfect interfaces have to be considered. Among all the imperfect interface models employed in the context of thermal conduction, the most widely used ones are the Kapitza's interface thermal resistance model (see, e.g. [1]), called also lowly conducting (LC) interface model, and the highly conducting (HC) interface model. The former stipulates that the normal component of the heat flux vector is continuous across an interface while the temperature across the interface suffers a jump proportional to the normal heat flux component. Viewed as dual to the former model, the latter

\footnotetext{
* Corresponding author. Tel.: +33(0) 1609577 97; fax: +33 (0) 160957799 .

E-mail addresses: hung.lequangđuniv-paris-estfr (H. Le Quang), pdchinhe imechacvn (D.C. Pham), guy.bonnetøuniv-paris-est.fr (G. Bonnet), qi-changheతf univ-paris-estff ( $Q-C$. He)
}

assumes that the temperature field is continuous across an interface while the normal heat flux is discontinuous across it. The LC and HC imperfect interface models were at the beginning proposed on the basis of some phenomenological considerations and next derived rigorously with the aid of an asymptotic approach. By considering a material surface or interface as the limit case of a very thin interphase situated between two bulk phases, the asymptotic approach showed that the Kapitza's interface thermal resistance model or the HC interface model is applicable according as the interphase is much less or more conducting than each of the constituents. Further, if the conductivity of the interphase is comparable with that of each of the phases, an imperfect interface model "intermediate" between the aforementioned "extreme" imperfect interface models is more appropriate. This approach, closely related to some mathematical techniques of homogenization, was initiated with the works $[2-8]$ in the general situation. A unified treatment of imperfect interfaces for multifield phenomena has been recently given by $\mathrm{Gu}$ and $\mathrm{He}[9]$.

The present work is concerned with the thermal conduction phenomenon in multiphase composites with LC and $\mathrm{HC}$ imperfect interfaces. The elaborated method and the results obtained for heat conduction are transposable to other transport phenomena like electric conduction, dielectrics, magnetism, diffusion and flow in porous media and to anti-plane elasticity, owing to their mathematical analogy and by means of appropriate physical interpretations. The composites studied in this work consist of a 


\begin{tabular}{|llll}
\hline \multicolumn{2}{l}{ Nomenclature } & & \\
HC & highly conducting & HSLB & Hashin-Shtrilaman lower bound \\
LC & lowly conducting & HSUB & Hashin-Shtrilman upper bound \\
RVE & representative volume element & BLB & Bruno lower bound \\
DD & dilute distribution & BUB & Bruno upper bound \\
MT & Mori-Tanaka & SCL & simple cubic lattice \\
SC & self-consistent & FCCL & face-centered cubic lattice \\
GSCA & generalized self-consistent approximation & BCCL. & body-centered cubic lattice \\
DA & differential approximation & FFT & fast Fourier transform \\
SLB & simple lower bound & ODF & orientation distribution function \\
SUB & simple upper bound & &
\end{tabular}

matrix in which circular inclusions or spherical inclusions of different sizes are, in the two-dimensional (2D) or three dimensional (3D) case, embedded via interfaces described by the LC or HC imperfect interface model. The objective of the present work is three-fold:

(i) First, it aims at extending the dilute distribution, MoriTanaka, self-consistent and generalized self-consistent schemes of micromechanics for incorporating the effect of imperfect interfaces in deriving the closed-form expressions for the effective conductivity and resistivity tensors of anisotropic multiphase composites. The present study is inspired from the recent works of Le Quang et al. $[10,11]$ in which the Eshelby's results and formalism for a circular or spherical inhomogeneity embedded in an elastic infinite matrix are extended to the thermal conduction phenomenon by accounting for the HC or LC imperfect interface between matrix and inclusions. Quite different from the relevan results of elasticity, Le Quang et al. $[10,11]$ showed that the generalized Eshelby's conduction tensor fields and localization tensor fields inside circular and spherical inhomogeneities remain uniform even in the presence of the $\mathrm{HC}$ or LC imperfect interface. Then, the analytical closed-form expressions for thermal effective conductivity of two-phase composites have been derived as functions of the interface properties and of the inhomogeneity size;

(ii) Second, it has the purpose of using the dilute solution results and the extended differential scheme to estimate the effective conductivity and resistivity tensors. We show that, in the presence of $\mathrm{HC}$ or LC imperfect interface, the effective conductivity and resistivity obtained by the differential approximation are comprised between the generalized lower and upper Hashin-Shtrikman bounds derived in [12-16];

(iii) Finally, it consists in studying the effects of LC and HC interfaces, inclusion size and orientation distribution of inclusions on the effective thermal properties of composites

The paper is structured as follows. In Section 2, the constitutive laws of the constituents of the composites under investigation the LC and HC imperfect interface models and the general form of the effective thermal conduction behavior arespecified. Section 3 is dedicated to deriving the size-dependent Eshelby's tensor fields in the context of thermal conduction in the presence of LC or HC imperfect interface. In Section 4, closed-form expressions are obtained for the effective conductivity by using the dilute, MoriTanaka, self-consistent, generalized self-consistent and differential schemes. In Section 5, the effects of LC and HC imperfect interfaces and inhomogeneity sizes on the effective conductivity of composites are numerically discussed and illustrated. In Section 6, a few concluding remarks are provided.

\section{Setting of the problem}

The composite (or multiphase material) under consideration consists typically of $N(\geqslant 1)$ inhomogeneities embedded in a matrix. The matrix and each inhomogeneity are assumed to be individually homogeneous. Relative to a Cartesian coordinate system $\left\{x_{1}, x_{2}, x_{3}\right\}$ in a right-handed orthonormal basis $\left\{\mathbf{f}_{1}, \mathbf{f}_{2}, \mathbf{f}_{3}\right\}$, the matrix, referred to as phase 0 , and the ith inhomogeneity, called phase $i$, have the linear thermal conduction behavior described by an anisotropic Fourier's law

$\mathbf{q}^{(p)}=\mathbf{K}^{(p)} \mathbf{e}^{(p)}$ or $\mathbf{e}^{(p)}=\mathbf{H}^{(p)} \mathbf{q}^{(p)}$,

where $q^{(p)}$ and $e^{(p)}$ are the heat flux and intensity fields of phase $p(=0,1,2, \ldots, N), \mathbf{K}^{(p)}$ and $\mathbf{H}^{(p)}=\left(\boldsymbol{K}^{(p)}\right)^{-1}$ stand for the second-order tensors of thermal conductivity and resistivity of phase $p$, which are symmetric, positive definite and in general orthotropic.

Let us denote by $\Omega$ the $d$-dimensional $(d=2$ or 3 ) domain occupied by a sample or a representative volume element (RVE) of the composite. The subdomains of $\Omega$ occupied by the matrix and the ith inhomogeneity are designated by $\omega^{(0)}$ and $\omega^{(i)}(i=1,2, \ldots, N)$. The interface between the matrix $\omega^{(\oplus)}$ and the ith inhomogeneity. denoted as $\Gamma^{(i)}$, is assumed to be imperfect. More precisely, the imperfect interface $\Gamma^{(i)}$ is described by either the HC or LC interface model.

First, according to the HC imperfect interface model (see, e.g., $[2,3,6,7,14-18])$, the interface $\Gamma^{(i)}$ between the matrix and ith inclusion is considered as a material surface of vanishing thickness across which the temperature field $T(\mathbf{x})$ is continuous. Thus, it follows from Hadamard's relation (see, e.g. [19]) that the tangential projection $\mathbf{e}^{5}(\mathbf{x})$ of the intensity field $\mathbf{e}(\mathbf{x})$ is continuous even though $\mathbf{e}(\mathbf{x})$ is generally discontinuous across $\Gamma^{(i)}$. By introducing the tangential projection operator $\mathbf{P}$ defined as

$\mathbf{P}(\mathbf{x})=\mathbf{I}-\mathbf{n}(\mathbf{x}) \otimes \mathbf{n}(\mathbf{x}), \quad \mathbf{x} \in \Gamma^{(i)}$,

with I standing for the second-order identity tensor and $\mathbf{n}(\mathbf{x})$ denoting the outward normal to $\Gamma^{(i)}$, the surface intensity field $\mathbf{e}^{3}(\mathbf{x})$ on $\Gamma^{(i)}$ can then be expressed as

$\mathbf{e}^{s}(\mathbf{x})=\mathbf{P}(\mathbf{x}) \mathbf{e}(\mathbf{x}), \quad \mathbf{x} \in \Gamma^{(i)}$.

The surface heat flux field $\mathbf{q}^{5}(\mathbf{x})=\mathbf{P}(\mathbf{x}) \mathbf{q}(\mathbf{x})$ is related to the surface intensity field $\mathbf{e}^{5}(\mathbf{x})$ by the following linear thermal isotropic law

$\mathbf{q}^{s}(\mathbf{x})=k_{s}^{(j)} \mathbf{e}^{s}(\mathbf{x})$,

where $\mathbf{x} \in \Gamma^{(i)}$ and $k_{s}^{(i)}$ is the surface thermal conductivity of $\Gamma^{(i)}$. Unlike the classical case where the interface is perfect, the normal component of the heat flux field $q(x)$ is in general discontinuous across the interface $\Gamma^{(i)}$ and its jump is connected to the surface heat flux field $\boldsymbol{q}^{s}(\mathbf{x})$ by the following surface energy conservation equation 
$\left(\mathbf{q}^{(0)}-\mathbf{q}^{(i)}\right) \cdot \mathbf{n}(\mathbf{x})=-\nabla_{s} \cdot \mathbf{q}^{s}(\mathbf{x}), \quad \mathbf{x} \in \Gamma^{(i)}$,

where $\nabla_{s} \cdot \mathbf{q}^{5}(\mathbf{x})$ represents the surface divergence of $\mathbf{q}^{5}(\mathbf{x})$. In particular, $\nabla_{s} \cdot \mathbf{q}^{s}(\mathbf{x})$ takes the form

$\nabla_{s} \cdot \mathbf{q}^{s}(\mathbf{x})=\frac{\partial q_{\theta}^{s}}{r \partial \theta}+\frac{q_{\theta}^{s}}{r} \cot \theta+\frac{1}{r \sin \theta} \frac{\partial q_{\varphi}^{s}}{\partial \varphi}$

in the spherical coordinate system $(r, \theta, \varphi)$ or

$\nabla_{s} \cdot \mathbf{q}^{s}(\mathbf{x})=\frac{\partial q_{\theta}^{s}}{r \partial \theta}$

in the polar coordinate system $(r, \theta)$.

Second, relative to the LC imperfect interface model (see, eg., $[5,7,12,13,17,18,20-22])$, the normal component of the heat flux $\mathbf{q}(\mathbf{x})$ is continuous across $\Gamma^{(i)}$, i.e. $\mathbf{q}^{(0)}(\mathbf{x}) \cdot \mathbf{n}(\mathbf{x})=q^{(i)}(\mathbf{x}) \cdot \mathbf{n}(\mathbf{x})$ for any $\mathbf{x} \in \Gamma^{(i)}$. At the same time, the temperature field $T(\mathbf{x})$ is in general discontinuous across $\Gamma^{(i)}$ and its jump is related to the normal component of the heat flux field by either one of the following interface conditions holding on $\Gamma^{(i)}$.

$T^{(0)}-T^{(i)}=-\alpha^{(j)}(\mathbf{n}) \mathbf{q}^{(0)} \cdot \mathbf{n}=-\alpha^{(i)}(\mathbf{n}) \mathbf{q}^{(i)} \cdot \mathbf{n}$,

$\beta^{(i)}(\mathbf{n})\left(T^{(0)}-T^{(i)}\right)=-\mathbf{q}^{(j)} \cdot \mathbf{n}=-\mathbf{q}^{(0)} \cdot \mathbf{n}$

where $\alpha^{(i)}$ and $\beta^{(i)}=1 / \alpha^{(i)}$ stand for the Kapitza thermal resistance and joint conductivity of $\Gamma^{(i)}$, respectively, and depend in general on $\mathbf{n}$.

Next, in order to clarify the physical background and the validity domain of $\mathrm{HC}$ and $\mathrm{LC}$ interface models used to describe $\Gamma^{(t)}$, we consider two configurations in Fig. 1. In the three-phase one, the inclusion phase $\omega^{(i)}$ is embedded into the matrix phase $\omega^{(0)}$ via the interphase $\omega^{(d)}$. According to the three-phase model (Fig. 1a), the interface between $\omega^{(i)}$ and $\omega^{(i)}$ and the one between $\omega^{(0)}$ and $\omega^{(c)}$ are assumed to be perfect, and the thickness $h^{(i)}$ of $\omega^{(c i)}$ is taken to be uniform and small in comparison with the size of $\omega^{(i)}$. In the second configuration (Fig. 1b), the interphase $\omega^{(d)}$ is now replaced by an imperfect interface of zero thickness $\Gamma^{(i)}$, situated at the middle surface of the interphase. The inclusion and matrix are then extended up to the middle surface $\Gamma^{(i)}$. By using an asymptotic expansion, the jump conditions that the imperfect interface $\Gamma^{(i)}$ has to verify for the two configurations to be physically equivalent within an error of order $0(h)$ were derived first by Sanchez-Palencia [2] and Pham Huy and Sanchez-Palencia [3] in a particular case and then completed by Miloh and Benveniste [6]. Hashin [7] and Benveniste [8] in the general situation. Gu and $\mathrm{He}[9]$ have recently obtained a unified imperfect interface model for multifield phenomena.

More precisely, when the interphase with thermal conductivity tensor $\mathbf{K}_{c}^{(i)}$ is assumed to be HC, or equivalently $\left\|\mathbf{K}_{c}^{(i)}\right\| \gg\left\|\mathbf{K}^{(t)}\right\|$ and $\left\|\mathbf{K}_{c}^{(i)}\right\| \gg\left\|\mathbf{K}^{(0)}\right\|$, then the conditions that the imperfect interface $\Gamma^{(i)}$ must satisfy are those of the HC interface model described above. The corresponding surface thermal conductivity tensor $\mathbf{K}_{s}^{(i)}$ of $\Gamma^{(i)}$ can be expressed in terms of $\mathbf{K}_{c}^{(i)}$ and $h^{(i)}$ as follows (see e.g. $[2,3]$ ) :

$\mathbf{K}_{s}^{(i)}=h^{(i)} \mathbf{S}_{t}^{(i)}$,

where

$\mathbf{S}_{t}^{(i)}=\mathbf{K}_{c}^{(i)}-\frac{\left(\mathbf{K}_{c}^{(i)} \mathbf{n}\right) \otimes\left(\mathbf{K}_{c}^{(i)} \mathbf{n}\right)}{\mathbf{K}_{c}^{(i)}:(\mathbf{n} \otimes \mathbf{n})}$.

In the present work, $\mathbf{K}_{c}^{(i)}$ is assumed to be circularly or spherically transverse isotropic (see e.g. $[23,24]$ ), i.e., $\mathbf{K}_{c}^{(i)}=k_{n}^{(i)}(\mathbf{n} \otimes \mathbf{n})+$ $k_{t}^{(j)}(\mathbf{I}-\mathbf{n} \otimes \mathbf{n})$ with $k_{t}^{(t)}$ and $k_{t}^{(/)}$standing for the radial and tangential thermal conductivities of the interphase and it is immediate from Eqs. (10) and (11) that

$\mathbf{K}_{\mathrm{s}}^{(i)}=k_{\mathrm{s}}^{(i)} \mathbf{P}, \quad k_{\mathrm{s}}^{(i)}=h^{(i)} k_{t}^{(i)}$,

In addition, due to the fact that the thermal conductivity tensor $\mathbf{K}_{c}^{*}$ of the interphase is positive definite, i.e. $k_{n}^{(i)}>0$ and $k_{t}^{(l)}>0$, it follows from Eq. (12) that $k_{s}^{(i)}$ is positive.

However, when the interphase with thermal conductivity tensor $\mathbf{K}_{c}^{(t)}$ is assumed to be LC, namely $\left\|\mathbf{K}_{c}^{(j)}\right\| \ll\left\|\mathbf{K}^{(i)}\right\|$ and $\left\|\mathbf{K}_{c}^{(0)}\right\| \ll\left\|\mathbf{K}^{(0)}\right\|$, then the conditions that the imperfect interface $\Gamma^{(i)}$ must satisfy are exactly those characterizing the imperfect interface model with Kapitza's thermal resistance described above. The corresponding Kapitza's thermal resistance of $\Gamma^{(i)}$ can be expressed in terms of $\mathbf{K}_{c}^{(i)}$ and $h^{(i)}$ as follows (see [8]):

$\alpha^{(i)}(\mathbf{n})=\frac{h^{(i)}}{\mathbf{n} \cdot \mathbf{K}_{c}^{(i)} \cdot \mathbf{n}}$.

In what follows, we make the assumption that $K_{c}^{(i)}$ is circularly or spherically orthotropic (see e.g. $[23,24]$ ), so that $k_{c}^{(i)}=\mathbf{n} \cdot \mathbf{K}_{c}^{(j)} \cdot \mathbf{n}$ is constant. Then, it is immediate from Eq. (13) that

$\alpha^{(i)}=\frac{h^{(i)}}{k_{c}^{(i)}}$

which is positive and independent of $\mathbf{n}$.

At the macroscopic scale, the composite under consideration is assumed to be homogeneous. Due to the linearity of the local constitutive laws of each phase and the imperfect interface $\Gamma^{(i)}$, the corresponding effective thermal conduction law remains linear and can be written as (see, e. g., [5-7])

$\mathbf{Q}=\mathbf{K}^{\text {eff }} \cdot \mathbf{E}$ or $\mathbf{E}=\mathbf{H}^{\text {eff }} \cdot \mathbf{Q}$.

where $\mathbf{K}^{\text {eff }}$ and $\mathbf{H}^{\text {eff }}=\left(\mathbf{K}^{\text {eff }}\right)^{-1}$ are the second-order tensors of effective thermal conductivity and resistivity, respectively, and the

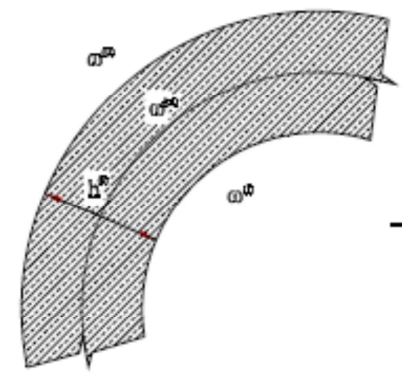

(a)

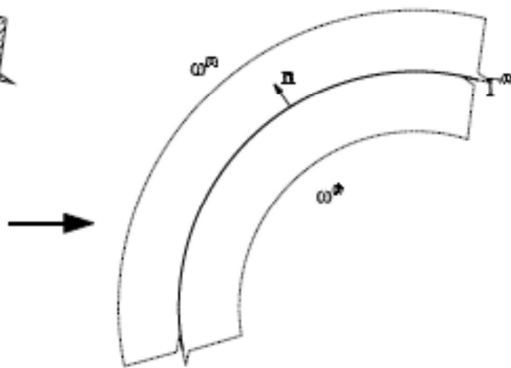

(a)

Fig. 1. Three- and two-phase configurations: (a) matrix/interphase/inclusion configuration; (b) matrix/inclusion configuration with imperfect interfaces. 
macroscopic heat flux $\mathbf{Q}$ and intensity field $\mathbf{E}$ over a representative volume element are defined by

$\mathbf{Q}=\frac{1}{|\Omega|} \int_{\Omega \Omega}(\mathbf{q} \cdot \boldsymbol{v}) \mathbf{x} d \mathbf{x}, \quad \mathbf{E}=-\frac{1}{|\Omega|} \int_{\Omega \Omega} T(\mathbf{x}) \boldsymbol{v}(\mathbf{x}) d \mathbf{x}$,

where $v(x)$ is the outward unit normal vector to $\partial \Omega$ and $|\Omega|$ denotes the volume or surface of $\Omega$ according as the $3 \mathrm{D}$ or $2 \mathrm{D}$ case is concerned.

However, due to the discontinuity of the normal heat flux field component across the HCinterface or due to the jump of the temperature field across the interface with Kapitza's thermal resistance, the macroscopic heat flux field $Q$ is given by (see [5-7])

$\mathbf{Q}=\langle\mathbf{q}\rangle+\frac{1}{|\Omega|} \sum_{i=1}^{N} \int_{\Gamma^{(j)}}\left(\mathbf{q}^{(0)} \cdot \mathbf{n}-\mathbf{q}^{(i)} \cdot \mathbf{n}\right) \mathbf{x} d \mathbf{x}$,

or equivalently by

$\mathbf{Q}=\langle\mathbf{q}\rangle-\frac{1}{|\Omega|} \sum_{i=1}^{N} \int_{\Gamma^{*}}\left(\nabla_{s} \cdot \mathbf{q}^{s}\right) \mathbf{x} d \mathbf{x}$

and the macroscopic intensity field $\mathbf{E}$ is determined by

$\mathbf{E}=\langle\mathbf{e}\rangle-\frac{1}{|\Omega|} \sum_{i=1}^{N} \int_{\Gamma^{(j)}}\left[T^{(0)}(\mathbf{x})-T^{(i)}(\mathbf{x})\right] \mathbf{n}(\mathbf{x}) d \mathbf{x}$,

or equivalently by

$\mathbf{E}=\langle\mathbf{e}\rangle+\frac{1}{|\Omega|} \sum_{i=1}^{N} \int_{\Gamma^{(x)}} \alpha^{(i)}\left[\mathbf{q}^{(i)}(\mathbf{x}) \cdot \mathbf{n}(\mathbf{x})\right] \mathbf{n}(\mathbf{x}) d \mathbf{x}$,

where $\langle\bullet\rangle$ is the volume or surface average of quantity $\bullet$ over the domain $\Omega$.

\section{Eshelby's conduction tensor field}

We consider now a 2D or 3D infinitely extended matrix domain $\Omega$ with the thermal conductivity and resistivity tensors $\mathbf{K}^{\circ}$ and $\mathbf{H}^{\circ}$. respectively. Let a heat-free intensity $\mathrm{e}^{\circ}$, which plays a role similar to the eigenstrain in elasticity, be uniformly distributed in a subdomain $\omega^{(i)}$ of $\Omega$ and vanish outside $\omega^{(i)}$. Introducing the characteris tic function $\chi^{(i)}(\mathbf{x})$ of $\omega^{(i)}$ by

$\chi^{(i)}(\mathbf{x})= \begin{cases}1 & \text { for } \mathbf{x} \in \omega^{(i)}, \\ 0 & \text { for } \mathbf{x} \notin \omega^{(i)},\end{cases}$

the prescribed heat-free intensity field $\mathbf{e}^{*}(\mathbf{x})$ can be expressed as

$\mathbf{e}^{*}(\mathbf{x})=\gamma^{(i)}(\mathbf{x}) \mathbf{e}^{0}$.

The interface $\Gamma^{(i)}$ between the inclusion $\omega^{(i)}$ and the external medium has the outward normal vector $\mathbf{n}$ and is assumed to be either $\mathrm{HC}$ or LC as has been described in Section 2.

When the subdomain $\omega^{(i)}$ is a spherical or circular inclusion of radius $R_{i}$, as proved in [10] for the case of $\mathrm{HC}$ imperfect interface and in [11] for the case of $L C$ imperfect interface, the intensity field solution $\mathbf{e}(\mathbf{x})$ remains uniform inside $\omega^{(i)}$. More precisely, the intensity field solution, both inside and outside $\omega^{(i)}$, is expressed as

$\mathbf{e}(\mathbf{x})=\overline{\mathbf{S}}(\mathbf{x}) \mathbf{e}^{0}$

where the tensor $\overline{\mathbf{S}}(\mathbf{x})$, called thereafter Eshelby's conduction tensor field, is defined as: $\overline{\mathbf{S}}(\mathbf{x})= \begin{cases}\mathbf{S}(\mathbf{x})-(d-1) \hat{k}_{s}^{(j)} \mathbf{S}(\mathbf{x}) \cdot \mathbf{H}^{0} \cdot \overline{\mathbf{S}}^{\oplus} & \text { for HC imperfect interface, } \\ \mathbf{S}(\mathbf{x})+\hat{\alpha}^{(i)} \mathbf{K}^{0}: \mathbf{M}(\mathbf{x}) \cdot \mathbf{K}^{0} \cdot\left[\overline{\mathbf{S}}^{\oplus}-\mathbf{I}\right] & \text { for LC imperfect interface, }\end{cases}$

where $\overline{\mathbf{S}}^{\omega}=\overline{\mathbf{S}}(\mathbf{x})$ with $\mathbf{x} \in \omega^{(i)}$ is the Eshelby's conduction tensor inside $\omega^{(i)}$ which is uniform and determined by

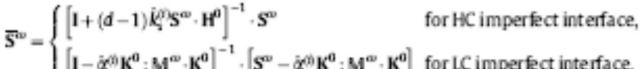

In Eqs. (24) and (25), $\hat{k}_{s}^{(h)}=k_{s}^{\left(\phi_{1}\right.} / R_{l}, \hat{\alpha}^{(t)}=\alpha^{(t)} / R_{l}, \mathrm{~S}(\mathbf{x})$ is the classical Eshelby's conduction tensor field over $\Omega$ without interface effect, $\mathbf{S}^{\omega}$ is the restriction of $\mathbf{S}(\mathbf{x})$ to $\omega^{(i)}$ and $M(\mathbf{x})$ and $M^{\omega}$ are two fourth-order tensors. The components $M_{m j m p}(\mathbf{x})$ of $M(\mathbf{x})$ and $S_{m i}(\mathbf{x})$ of $\mathbf{S}(\mathbf{x})$ can be calculated by (see [11])

$M_{m, m p}(\mathbf{x})=-\frac{\partial N_{m, p}(\mathbf{x})}{\partial x_{m}}+\delta_{n p} D_{m j}(\mathbf{x}), \quad S_{m i}(\mathbf{x})=D_{m j}(\mathbf{x}) K_{j i}^{0}$,

with

$N_{n, p}(\mathbf{x})=\frac{\partial^{2}}{\partial x_{n} \partial x_{j}} \int_{\omega^{\hat{\theta}}} G(\mathbf{y}-\mathbf{x}) y_{p} d \mathbf{y}$,

$D_{m j}(\mathbf{x})=\frac{\partial^{2}}{\partial x_{m} \partial x_{j}} \int_{\omega^{\omega}} G(\mathbf{y}-\mathbf{x}) d \mathbf{y}$,

where $G$ is the Green function of thermal conduction.

It is shown in the Appendix that when $\mathbf{x} \in \omega^{(i)}$, then $N_{i, p o}(\mathbf{x})$ and $D_{m j}(\mathbf{x})$ are polynomial functions of degree 1 and 0 in terms of the position vector $\mathbf{x}$. Thus, the tensor $M(\mathbf{x})$ with the components $M_{\text {nimp }}(\mathbf{x})$ given by Eq. (26) is uniform inside $\omega^{(i)}$ and equal to a constant tensor $M^{\omega}$, i.e. $M(x)=M^{\omega}$ when $\mathbf{x} \in \omega^{(i)}$.

From a dual point of view, we now consider the conjugate Eshelby's conduction problem where a heat flux is uniformly distributed within a spherical or circular inclusion, or equivalently $\mathbf{q}^{*}(\mathbf{x})=\chi^{(i)}(\mathbf{x}) \mathbf{q}^{0}$. The general heat flux field solution, both inside and outside the inclusion $\omega^{(i)}$, can be determined via the conjugate Eshelby's conduction tensor field with HC or LC imperfect interface, denoted by $\overline{\mathbf{C}}(\mathbf{x})$, such as

$\mathbf{q}(\mathbf{x})=\overline{\mathbf{C}}(\mathbf{x}) \cdot \mathbf{q}^{0}$.

It can be shown that $\overline{\mathbf{C}}(\mathbf{x})$ is related to $\overline{\mathbf{S}}(\mathbf{x})$ by

$\overline{\mathbf{C}}(\mathbf{x})=-\mathbf{K}^{0} \cdot \overline{\mathbf{S}}(\mathbf{x}) \cdot \mathbf{H}^{0}+\chi^{i)}(\mathbf{x}) \mathbf{I}$

This equation indicates that, as expected, the conjugate Eshelby's conduction tensor $\overline{\mathbf{C}}(\mathbf{x})$ with HC or LC imperfect interface is also uniform inside $\omega^{(i)}$, i.e. $\overline{\mathbf{C}}(\mathbf{x})=\overline{\mathbf{C}}^{\infty}$ for $\mathbf{x} \in \omega^{(i)}$ with

$\overline{\mathbf{C}}^{\omega}=-\mathbf{K}^{0} \cdot \overline{\mathbf{S}}^{\omega} \cdot \mathbf{H}^{0}+\mathbf{I}$.

In particular, when the interface between the ith inclusion and the exterior medium is perfect, i.e. $\alpha^{(i)}=0, k_{s}^{(j)}=0$ or when the size $R_{i}$ of the inclusion $\omega^{(i)}$ is very large, we obtain from Eq. (30) the classical conjugate Eshelby's conduction tensor

$\mathbf{C}(\mathbf{x})=-\mathbf{K}^{0} \cdot \mathbf{S}(\mathbf{x}) \cdot \mathbf{H}^{0}+\chi^{(i)}(\mathbf{x}) \mathbf{I}$

and its restriction to $\omega^{(i)}$

$\mathbf{C}^{\omega}=-\mathbf{K}^{0} \cdot \mathbf{S}^{\omega} \cdot \mathbf{H}^{0}+\mathbf{I}$

We recall that the previous properties of the Eshelby's conduction tensor field $\overline{\mathbf{S}}(\mathbf{x})$ with $\mathrm{HC}$ or LC imperfect interface hold for any thermal anisotropy of the material forming $\Omega$. In order to obtain the explicit expression of $\overline{\mathbf{S}}(\mathbf{x})$, we now consider the most important special case where the 2D or 3D infinite body $\Omega$ consists of an isotropic material whose thermal conductivity tensor takes the form $K^{0}=k_{0} \mathbf{I}$ with $k_{0}$ being a positive scalar. Correspondingly, the expressions of the Eshelby's conduction tensor field $\overline{\mathbf{S}}(\mathbf{x})$ and of the restriction of $\overline{\mathbf{S}}(\mathbf{x})$ to $\omega^{(i)}$ take the simple forms

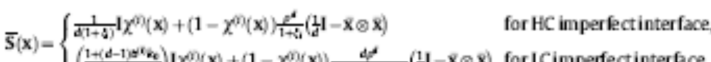

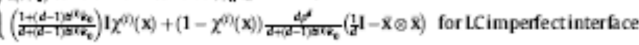

and 


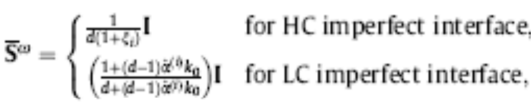

where

$\rho=\frac{R}{r}=\frac{R}{|\mathbf{X}|}, \quad \overline{\mathbf{x}}=\frac{\mathbf{x}}{r}, \quad \xi_{i}=\frac{(d-1)}{d} \frac{\hat{k}_{s}^{(i)}}{k_{0}}$.

Similarly, we obtain the expressions for the conjugate Eshelby's conduction tensors with $\mathrm{HC}$ and LC imperfect interface effects:

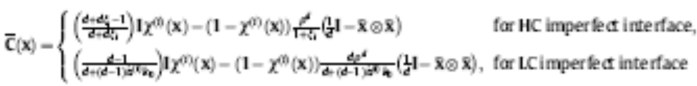

and

$\overline{\mathbf{C}}^{\omega}= \begin{cases}\left(\frac{d+d g_{j}-1}{d+d L_{i}}\right) \mathbf{I} & \text { for HC imperfect interface, } \\ \left(\frac{d-1}{d+(d-1) \bar{\alpha}^{n} k_{1}}\right) \mathbf{I} & \text { for LC imperfect interface. }\end{cases}$

As mentioned above, in the particular case where the interface between the ith inclusion and the exterior medium is perfect or when the size $R_{i}$ of the inclusion $\omega^{(i)}$ is so large that $\hat{\alpha}^{(i)}=\alpha^{(i)} / R_{i}$ and $\hat{k}_{s}^{(i)}=k_{s}^{(j)} / R_{1}$ tend to zero, the classical Eshelby's conduction tensors, $\mathbf{S}(\mathbf{x})$ and $\mathbf{C}(\mathbf{x})$, as well as its restrictions to $\omega^{(i)}, \mathbf{S}^{m}$ and $\mathbf{C}^{m}$, for an isotropic material can be obtained from Eqs. (34)-(38) by setting $\hat{\alpha}^{(j)}=0$ and $\hat{k}_{\hat{s}}^{(t)}=0$ :

$\mathbf{S}(\mathbf{x})=\frac{1}{d} \mathbf{I} \chi^{(i)}(\mathbf{x})+\left(1-\chi^{(j)}(\mathbf{x})\right) \rho^{d}\left(\frac{1}{d} \mathbf{I}-\overline{\mathbf{x}} \otimes \overline{\mathbf{x}}\right)$,

$\mathbf{C}(\mathbf{x})=\left(\frac{d-1}{d}\right) \mathbf{I} \chi^{(j)}(\mathbf{x})+\left(1-\chi^{(i)}(\mathbf{x})\right) \rho^{d}\left(\frac{1}{d} \mathbf{I}-\overline{\mathbf{x}} \otimes \overline{\mathbf{x}}\right)$,

$\mathbf{S}^{\omega}=\frac{1}{d} \mathbf{I}, \quad \mathbf{C}^{\omega}=\left(\frac{d-1}{d}\right) \mathbf{I}$

where $\rho$ and $\overline{\mathbf{x}}$ are defined by Eq. (36).

\section{Estimations for effective conductivity and resistivity}

4.1. Dilute, Mori-Tanaka, seif-consistent and generalized selfconsistent modeis

The closed-form expressions obtained in this Section for the effective thermal conductivity and resistivity tensors extend the relevant results of [10,11] to the multiphase composites with $\mathrm{HC}$ and LC imperfect interfaces. This generalization is carried out by using the preliminary results of Sections 2 and 3, and by applying the dilute distribution, Mori-Tanaka, self-consistent and generalized self-consistent models in micromechanics. The final results obtained for the effective thermal conductivity and resistivity tensors are summarized and discussed below. For briefness, their derivation and the corresponding technical details are not given here and the reader can refer to $[10,11]$

First, for the composite with HC imperfect interfaces, the effective thermal conductivity tensor is estimated according to the aforementioned micromechanics models as follows:

- When the dilute distribution (DD) model is used, we have

$$
\mathbf{K}^{D D}=\mathbf{K}^{0}+\sum_{i=1}^{N} c_{i} \mathbf{A}\left(\mathbf{K}^{0}, \mathbf{K}^{(i)}, \hat{k}_{s}^{(i)}\right) \text {. }
$$

Here and thereafter, $c_{p}$ denotes the volume or surface fraction of phase $p$ and

$$
\begin{aligned}
& \mathbf{A}\left(\mathbf{K}^{0}, \mathbf{K}^{(i)}, \hat{k}_{s}^{(j)}\right)=\left[\mathbf{K}^{(i)}-\mathbf{K}^{0}+(d-1) \hat{k}_{s}^{(t)} \mathbf{1}\right] \cdot \mathbf{D}\left(\mathbf{K}^{0}, \mathbf{K}^{(i)}, \hat{k}_{s}^{(j)}\right), \\
& \mathbf{D}\left(\mathbf{K}^{0}, \mathbf{K}^{(i)}, \hat{k}_{s}^{(i)}\right)=\left[\mathbf{S}^{\infty} \cdot\left(\overline{\mathbf{S}}^{\infty}\right)^{-1}+\mathbf{S}^{\infty} \cdot \mathbf{H}^{0} \cdot\left(\mathbf{K}^{(i)}-\mathbf{K}^{0}\right)\right]^{-1} .
\end{aligned}
$$

In particular, when the matrix and ith inclusion phases are isotropic and have the thermal conductivities $k_{0}$ and $k_{1}$, respectively, and by assuming that the effective behavior is also isotropic, the effective thermal conductivity is reduced to

$$
k^{D D}=k_{0}+\sum_{i=1}^{N} c_{i} a\left(k_{0}, k_{i}, \hat{k}_{s}^{(i)}\right)
$$

with

$$
a\left(k_{0}, k_{i}, \hat{k}_{s}^{(i)}\right)=\frac{d k_{0}\left[k_{i}-k_{0}+(d-1) \hat{k}_{s}^{(i)}\right]}{k_{i}+(d-1)\left(k_{0}+\hat{k}_{s}^{(j)}\right)} .
$$

- When the Mori-Tanala (MT) model is adopted, we obtain

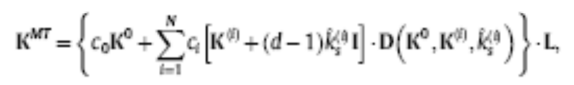

where

$$
\mathbf{L}=\left\{\sum_{i=1}^{N} c_{i} \mathbf{D}\left(\mathbf{K}^{0}, \mathbf{K}^{(j)}, \hat{k}_{s}^{(j)}\right)+c_{0} \mathbf{I}\right\}^{-1} .
$$

The corresponding effective thermal conductivity of the isotropic composite with an isotropic matrix and $N$ isotropic inclusion phases characterized by the thermal conductivities $k_{0}$ and $k_{a}$ is given by

$$
k^{M T}=k_{0}+\frac{\sum_{i=1}^{N} c_{i} D_{i}\left[k_{i}-k_{0}+(d-1) \hat{k}_{s}^{(i)}\right]}{c_{0}+\sum_{i=1}^{N} c_{i} D_{i}}
$$

with

$$
D_{i}=\frac{d k_{0}}{k_{i}+(d-1)\left(k_{0}+\hat{k}_{s}^{(i)}\right)} .
$$

- If the self-consistent (SC) model is applied, we get

$$
\mathbf{K}^{s c}=\mathbf{K}^{0}+\sum_{i=1}^{N} c_{i}\left[\mathbf{K}^{(t)}-\mathbf{K}^{0}+(d-1) \hat{k}_{s}^{(i)} \mathbf{I}\right] \cdot \mathbf{D}\left(\mathbf{K}^{s c}, \mathbf{K}^{(i)}, \hat{k}_{s}^{(a)}\right) .
$$

This is an implicit equation for the effective conductivity tensor $\mathbf{K}^{S C}$. When the matrix and inclusion phases are isotropic and characterized by the thermal conductivities $k_{0}$ and $k_{i}$ and when the effective behavior is also isotropic, the effective thermal conductivity is determined as the positive real root of the following equation

$$
k^{\mathscr{C}}=k_{0}+\sum_{i=1}^{N} \frac{c_{i} d k^{\mathscr{C}}\left[k_{i}-k_{0}+(d-1) \hat{k}_{s}^{(i)}\right]}{k_{i}+(d-1)\left(k^{S C}+\hat{k}_{s}^{(i)}\right)} .
$$

- Applying the generalized self-consistent approximation (GSCA) model, we have

$$
\begin{aligned}
k^{C S A} & =k_{0}+\frac{c_{1} d k_{0}\left[k_{1}-k_{0}+(d-1) \hat{k}_{s}^{(1)}\right]}{d k_{0}+\left(1-c_{1}\right)\left[k_{1}-k_{0}+(d-1) \hat{k}_{s}^{(1)}\right]} \\
& \equiv k^{\mathrm{M} T}
\end{aligned}
$$

for the isotropic two-phase composite with $k_{0}$ and $k_{1}$ being the conductivities of the matrix and inclusion phases, respectively. We recall that with GSCA model, it is only in the case where the matrix and inclusion phases are all isotropic that the estimation of the effective thermal conductivity can be obtained. Moreover, by assuming that the inclusion phase is more conducting than the matrix phase, it is interesting to notice that the expression (53) with $d=3$ for the effective conductivity based on MT or GSCA scheme 
coincides exactly with the generalized Hashin-Shtrikman lower bound and Bruno lower bound established by Lipton [14,15], Lipton and Talbot [16] when including imperfect interface effects.

Second, for the composite with LC imperfect interfaces, the effective thermal resistivity tensor is provided by:

- the dilute distribution (DD) model as

$$
\mathbf{H}^{D D}=\mathbf{H}^{0}+\sum_{i=1}^{N} c_{i} \mathbf{B}\left(\mathbf{H}^{0}, \mathbf{H}^{(i)}, \hat{\alpha}^{(i)}\right),
$$

where

$$
\begin{aligned}
& \mathbf{B}\left(\mathbf{H}^{0}, \mathbf{H}^{(i)}, \hat{\alpha}^{(i)}\right)=\left[\mathbf{H}^{(i)}-\mathbf{H}^{0}+\hat{\alpha}^{(i)} \mathbf{I}\right] \cdot \mathbf{F}\left(\mathbf{H}^{0}, \mathbf{H}^{(i)}, \hat{\alpha}^{(i)}\right), \\
& \mathbf{F}\left(\mathbf{H}^{0}, \mathbf{H}^{(i)}, \hat{\alpha}^{(i)}\right)=\left[\mathbf{C}^{(j)} \cdot\left(\overline{\mathbf{C}}^{\omega}\right)^{-1}+\mathbf{C}^{(i)} \cdot \mathbf{K}^{0} \cdot\left(\mathbf{H}^{(i)}-\mathbf{H}^{0}\right)\right]^{-1} \cdot
\end{aligned}
$$

In the particular case where matrix and inclusion phases are all isotropic with the thermal resistivities $h_{0}$ and $h_{i}$, respectively, and by assuming that the effective behavior is also isotropic, the effective thermal resistivity is provided by

$$
h^{D D}=h_{0}+\sum_{i=1}^{N} c_{i} b\left(h_{0}, h_{i}, \hat{\alpha}^{(i)}\right),
$$

with

$$
b\left(k_{0}, k_{i}, \hat{\alpha}^{(i)}\right)=\frac{d h_{0}\left[h_{i}-h_{0}+\hat{\alpha}^{(i)}\right]}{h_{0}+(d-1)\left(h_{i}+\hat{\alpha}^{(i)}\right)} .
$$

- the Mori-Tanaka (MT) model as

$$
\mathbf{H}^{\mathrm{M} T}=\left\{c_{0} \mathbf{H}^{0}+\sum_{i=1}^{N} c_{i}\left[\mathbf{H}^{(j)}+\hat{\alpha}^{(i)} \mathbf{I}\right] \cdot \mathbf{F}\left(\mathbf{H}^{0}, \mathbf{H}^{(i)}, \hat{\alpha}^{(i)}\right)\right\} \cdot \mathbf{M},
$$

where

$$
\mathbf{M}=\left\{\sum_{i=1}^{N} c_{i} \mathbf{F}\left(\mathbf{H}^{0}, \mathbf{H}^{(i)}, \hat{\alpha}^{(i)}\right)+c_{0} \mathbf{I}\right\}^{-1} .
$$

The corresponding effective thermal resistivity of the isotropic composite made of an isotropic matrix and of $N$ isotropic inclusion phases whose thermal resistivities are $h_{0}$ and $h_{i}$, respectively, is given by

$$
h^{M T}=h_{0}+\frac{\sum_{i=1}^{N} c_{i} F_{i}\left[h_{i}-h_{0}+\hat{\alpha}^{(j)}\right]}{c_{0}+\sum_{j-1}^{N} c_{i} F_{i}},
$$

where

$$
F_{i}=\frac{d h_{0}}{h_{0}+(d-1)\left(h_{i}+\hat{\alpha}^{(i)}\right)} .
$$

- the self-consistent (SC) model as

$$
\mathbf{H}^{S C}=\mathbf{H}^{0}+\sum_{i=1}^{N} c_{i}\left[\mathbf{H}^{(i)}-\mathbf{H}^{0}+\hat{\alpha}^{(i)} \mathbf{I}\right] \cdot \mathbf{F}\left(\mathbf{H}^{S C}, \mathbf{H}^{(i)}, \hat{\alpha}^{(i)}\right) .
$$

This tensor equation al lows to obtain the effective resistivity tensor $\mathbf{H}^{\mathrm{SC}}$. However, if the matrix and inclusion phases are all isotropic and characterized by the thermal resistivities $h_{0}$ and $h_{i}$ and if the effective behavior is assumed to be also isotropic, Eq. (63) reduces

$$
h^{S C}=h_{0}+\sum_{i=1}^{N} \frac{c_{i} d h^{S C}\left[h_{i}-h_{0}+\hat{\alpha}^{(i)}\right]}{h^{S C}+(d-1)\left(h_{i}+\hat{\alpha}^{(i)}\right)}
$$

and the effective thermal resistivity $h^{s c}$ is determined as the positive real root of Eq. (64).

- the generalized self-consistent approximation (GSCA) model as

$$
h^{\omega C A}=h_{0}+\frac{c_{i} d h_{0}\left[h_{1}-h_{0}+\tilde{\alpha}^{(1)}\right]}{h_{0} c_{1} d+\left(1-c_{1}\right)\left[(d-1)\left(h_{1}+\hat{\alpha}^{(1)}\right)+h_{0}\right]} \equiv h^{M r}
$$

for the case where the isotropic composite constituting of only two isotropic phases with $h_{0}$ and $h_{1}$ denoting the resistivities of the matrix and inclusion phases, respectively. Moreover, it is easy to check that the corresponding effective conductivity obtained by inverting the effective resistivity given by (65) with $d=3$ is identical to the generalized Hashin-Shtrikman upper bound and Bruno upper bound of two-phase composite containing the inhomogeneities less conducting than the matrix phase (see, e. g., $[12,13,16]$ ).

\section{Differential approximation model (DA)}

In this section, the derivation of the closed-form expressions for the effective thermal conductivity and resistivity tensors of composites with $\mathrm{HC}$ and LC imperfect interfaces is carried out by applying the differential scheme construction process [25-27]. According to this construction process, the composite described in Section 2 with $N$ inhomogeneities embedded in a matrix phase is built by introducing progressively an infinitesimal volume fraction of $N$ inhomogeneities into the host medium which is initially homoge neous and corresponds to the matrix phase. More precisely, at each step of this procedure, we add proportionally infinitesimal volume amounts $c_{i} \Delta t(\Delta t \ll 1, i=1,2, \ldots, N)$ of randomly circular or spherical inclusions into the composite having been al ready constructed at the previous step, which contains volume fractions $c_{t} t$ of the inclusion phases. Thus, during the construction process, the dimensionless model parameter $t$ increases from 0 to 1 . By assuming that the effective thermal conductivity and resistivity tensors of the already constructed composite of the previous step are $\mathbf{K}(t)$ and $\mathbf{H}(t)$, respectively, and by applying the dilute solutions presented above in Eqs. (42) and (54), the effective thermal conductivity and resistivity tensors of the new composite are given by

$\mathbf{K}(t+\Delta t)=\mathbf{K}(t)+\mathcal{D} K(t)$

$$
=\mathbf{K}(t)+\sum_{i=1}^{N} \frac{c_{i} \Delta t}{1+c_{l} \Delta t} \mathbf{A}\left(\mathbf{K}(t), \mathbf{K}^{(i)}, \hat{k}_{s}^{(i)}\right)
$$

for the case of composite with HC imperfect interface and

$\mathbf{H}(t+\Delta t)=\mathbf{H}(t)+\mathcal{D H}(t)$

$$
=\mathbf{H}(t)+\sum_{i=1}^{N} \frac{c_{i} \Delta t}{1+c_{l} \Delta t} \mathbf{B}\left(\mathbf{H}(t), \mathbf{H}^{(i)}, \hat{\alpha}^{(i)}\right)
$$

for the case of composite with LC imperfect interface.

In Eqs. (66) and (67), D • denotes a differential of $\bullet ; c_{l}$ designs the total volume fractions of all inclusion phases, i.e. $c_{l}=\sum_{l=1}^{N} c_{l}$ and $\frac{q_{A}}{1+c M t}$ stands for the newly added volume fraction of ith inclusion phase. Thus, the volume fraction of the ith inclusion phase increases by

$c_{i} D t=\frac{c_{i} t+c_{i} \Delta t}{1+c_{l} \Delta t}-c_{i} t=\frac{c_{i} \Delta t}{1+c_{l} \Delta t}\left(1-c_{l} t\right)$.

By combining the last equation with (66) and (67), we obtain the following differential equations for the effective thermal conductivity and resistivity tensors, $\mathbf{K}^{D A}$ and $\mathbf{H}^{D A}$, of multiphase materials with $\mathrm{HC}$ and $\mathrm{LC}$ imperfect interfaces:

$$
\frac{\mathcal{D K}(t)}{\mathcal{D} t}=\frac{1}{1-c_{l} t} \sum_{i=1}^{N} c_{i} \mathbf{A}\left(\mathbf{K}(t), \mathbf{K}^{(i)}, \hat{k}_{\Sigma}^{(i)}\right), \quad \mathbf{K}(0)=\mathbf{K}^{0}, \mathbf{K}^{D A}=\mathbf{K}(1),
$$

$$
\frac{D \mathbf{H}(t)}{\mathcal{D} t}=\frac{1}{1-c_{l} t} \sum_{i=1}^{N} c_{i} \mathbf{B}\left(\mathbf{H}(t), \mathbf{H}^{(i)}, \hat{\alpha}^{(i)}\right), \quad \mathbf{H}(0)=\mathbf{H}^{0}, \quad \mathbf{H}^{D A}=\mathbf{H}(1) .
$$

In the important particular case where the matrix and inclusion phases are all isotropic and have the thermal conductivities (or 
resistivities) $k_{0}$ and $k_{i}$ (or $h_{0}$ and $h_{i}$ ), respectively, and by assuming that the effective behavior is also isotropic, the differential Eqs. (69) and (70) for the effective thermal conductivity and resistivity. $k^{D A}$ and $h^{D A}$, of the multiphase composite reduce to

$$
\begin{aligned}
& \frac{\mathcal{D} k(t)}{\mathcal{D} t}=\frac{1}{1-c_{l} t} \sum_{i=1}^{N} c_{i} a\left(k, k_{i}, k_{s}^{D(j)}\right), k(0)=k_{0}, k^{D A}=k(1), \\
& \frac{\mathcal{D} h(t)}{\mathcal{D} t}=\frac{1}{1-c_{l} t} \sum_{i=1}^{N} c_{i} b\left(h, h_{i}, \hat{\alpha}^{(j)}\right), \quad h(0)=h_{0}, \quad h^{D A}=h(1)
\end{aligned}
$$

for the cases of composites with HC and LC imperfect interfaces.

Further, for a two-phase composite, the differential Eqs. (71) and (72) can be specified, respectively, as

$$
\begin{array}{ll}
\frac{D k(t)}{D t}=\frac{d c_{l}\left[(d-1) \hat{k}_{s}^{(i)}+k_{i}-k\right] k}{\left(1-c_{l} t\right)\left[(d-1)\left(\hat{k}_{s}^{(i)}+k\right)+k_{i}\right]}, & k(0)=k_{0}, \\
\frac{\mathcal{D} h(t)}{\mathcal{D} t}=\frac{d c_{l}\left[\hat{\alpha}^{(i)}+h_{i}-h\right] h}{\left(1-c_{l} t\right)\left[(d-1)\left(\hat{\alpha}^{(i)}+h_{i}\right)+h\right]}, \quad h(0)=h_{0} .
\end{array}
$$

By solving Eqs. (73) and (74), we obtain the following explicit formulae for the two-phase composite

- when the imperfect interfaces are HC,

$$
k^{D A}= \begin{cases}k_{i}+2 \hat{k}_{s}^{(i)}-\frac{1}{6} \gamma_{1} \gamma_{2}+\frac{2 \gamma_{1}^{2}}{\eta_{2}} & \text { for case 3D, } \\ k_{i}+\hat{k}_{s}^{(i)}+\frac{1}{2} \beta_{1}+\frac{1}{2} \beta_{2} \sqrt{4 \beta_{1} \hat{k}_{s}^{(i)}+\beta_{1}^{2}+4 \beta_{1} k_{i}} & \text { for case 2D, }\end{cases}
$$

with

$$
\begin{aligned}
& \gamma_{1}=\frac{c_{0}\left(2 k_{3}^{(9)}+k_{1}-k_{0}\right)}{k_{0}^{1 / 3}},
\end{aligned}
$$

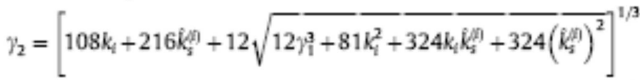

$$
\begin{aligned}
& \beta_{1}=\frac{c_{0}^{2}\left(k_{s}^{(4)}+k_{i}-k_{0}\right)^{2}}{k_{0}}, \quad \beta_{2}=\operatorname{sgn}\left(k_{0}-k_{i}-\hat{k}_{s}^{\prime \prime}\right),
\end{aligned}
$$

where $\operatorname{sgn}(x)$ is defined as $\operatorname{sgn}(x)=1$ if $x>0, \operatorname{sgn}(x)=0$ if $x=0$ and $\operatorname{sgn}(x)=-1$ if $x<0$

- when the imperfect interfaces are LC,

$$
h^{D A}= \begin{cases}h_{i}+\hat{\alpha}^{(i)}-\frac{1}{5}\left(\frac{1}{2} \gamma_{4}+2 \frac{\gamma_{1}^{2}}{\gamma_{4}}-\gamma_{3}\right)^{2} \gamma_{3} & \text { for case 3D, } \\ h_{i}+\hat{\alpha}^{(i)}+\frac{1}{2} \beta_{3}+\frac{1}{2} \beta_{4} \sqrt{4 \beta_{3} \hat{\alpha}^{(i)}+\beta_{3}^{2}+4 \beta_{3} k_{i}} & \text { for case 2D, }\end{cases}
$$

with

$$
\begin{aligned}
\gamma_{3}= & \frac{c_{0}\left(\hat{\alpha}^{(9)}+h_{i}-h_{0}\right)}{h_{0}^{2 / 3}}, \\
\gamma_{4}= & {\left[108 h_{i}+108 \hat{\alpha}^{(\hat{9}}-8 \gamma_{3}^{3}\right.} \\
& \left.+12 \sqrt{81 h_{i}^{2}+162 h_{i} \hat{\alpha}^{(9)}-12 h_{i} \gamma_{3}^{3}+81\left(\hat{\alpha}^{(i)}\right)^{2}-12 \hat{\alpha}^{(i)} \gamma_{3}^{3}}\right]^{1 / 3}, \\
\beta_{3}= & \frac{c_{0}^{2}\left(\hat{k}_{i}^{4}+k_{i}-k_{0}\right)^{2}}{k_{0}}, \quad \beta_{4}=\operatorname{sgn}\left(h_{0}-h_{i}-\hat{\alpha}^{(0)}\right) .
\end{aligned}
$$

Both the generalized self-consistent approximation (GSCA) and differential approximation (DA) become exact results for some particular microstructures: the hierarchical one constructed incrementally for DA, and the Hashin-Shtrikman polydisperse coated sphere assemblage for GSCA (see, e.g. [12-16]). This fact secures that these two approximations comply with certain mathematical requirements: they never violate bounds or exact mathematical restrictions imposed upon an effective property. Both models are idealistic and involve inhomogeneities down to infinitesimal sizes (while the relations $\hat{k}_{\mathrm{s}}^{(d)}=k_{s}^{\left(d_{1}\right.} / R_{1}$ or $\hat{\alpha}^{(d)}=\alpha^{(d)} / R_{1}$ are fixed in the models), but intend to approximate practical mixtures with some overall characteristics ( $\hat{k}_{s}^{(t)}$ or $\hat{\alpha}^{(i)}$ among others, in particular)

\section{Numerical applications}

The estimations established in the previous Section 4 for the effective thermal conductivity and resistivity of multiphase mate rials whose interfaces are described by either the HC or LC imperfect interface model are now numerically illustrated by considering the first example where the mono-sized spherical isotropic inhomogeneities of conductivity $k_{i}$, resistivity $h_{i}=1 / k_{i}$ and radius $R$ are introduced into a host isotropic matrix phase of conductivity $k_{0}$ and resistivity $h_{0}=1 / k_{0}$. Moreover, to study the effect of imperfect interfaces on the effective thermal conductivity of the resulting composite, two additional cases with inclusion phase being more and less conducting than the matrix phase are investigated. The normalized effective thermal conductivity provided in Section 4 with the differential approximation (DA) model as well as with the dilute distribution (DD), Mori-Tanala (MT), self-consistent (SC) and generalized self-consistent approximation (GSCA) models are plotted versus the inhomogeneity volume fraction $c_{i}$ in Fig. 2 for the case where the interfaces between the matrix and inclusion phases are $\mathrm{HC}$ imperfect interfaces and in Fig. 3 for the case where the interfaces between the matrix and inclusion phases are IC imperfect interfaces. These estimations for the effective thermal conductivity are then compared in Figs. 2 and 3 with the corre sponding simplest lower and upper bounds (SLB and SUB), generalized Hashin-Shtrikman lower and upper bounds (HSLB and HSUB) and Bruno lower and upper bounds (BLB and BUB) of the effective thermal conductivity. For more details about these bounds of the effective thermal conductivity of composite with $\mathrm{HC}$ and LC imperfect interfaces, the reader can refer to [12-16,28]. For another comparison, the estimations obtained by Cheng and Torquato $[18,21]$ for the effective thermal conductivities of composites consisting of periodic arrays of spherical inclusions such as simple cubic lattice $(\mathrm{SCL})$, face-centered cubic lattice (FCCL) and body-centered $\mathrm{Cu}$ bic lattice (BCCL) are also presented in Figs. 2 and 3.

Similarly, when the inhomogeneity phase is less conducting than the matrix phase (case 2), the normalized effective thermal conduc-

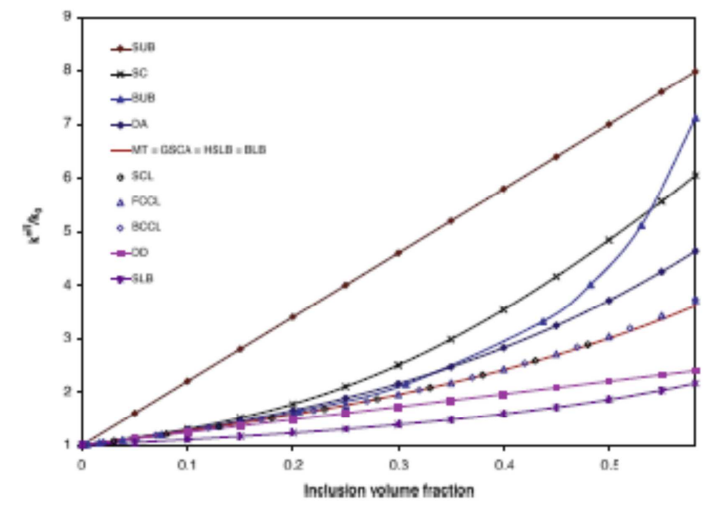

Fig. 2. Estimations and bounds of the effective thermal conductivity versus the inhomogeneity volume fraction of the two-phase composite with HC imperfect interfaces, $k / k_{0}-5$ and $k^{2} / k_{0}=4$. 


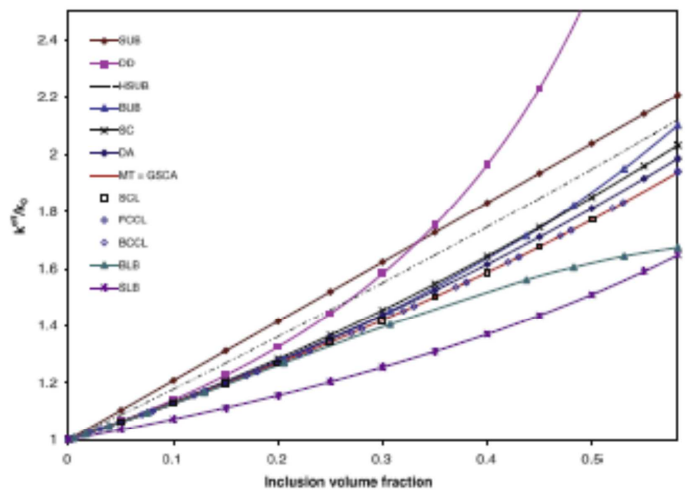

Fig. 3. Estimations and bounds of the effective thermal conductivity versus the inhomogeneity volume fraction of the two-phase composite with LC imperfect interfaces, $k_{i} / k_{0}=5$ and $\hat{\alpha}^{2} / h_{0}=0.125$

tivity values obtained from the differential approximation (DA), dilute distribution (DD), Mori-Tanaka (MT), self-consistent (SC) and generalized self-consistent approximation (GSCA) schemes are shown first in Figs. 4 and 5 and then compared with its simplest. generalized Hashin-Shtrikman and Bruno bounds as well as with the effective thermal conductivities of the periodic composites of simple, body-centered and face-centered cubic arrays.

It is seen from Figs. $2-5$ that:

(i) the estimations for the effective thermal conductivity derived by the micromechanical models described in Section 4 , except the DD scheme, are situated between the simplest bounds as well as the generalized Hashin-Shtrikman bounds. However, these estimations for the effective thermal conductivity are not always comprised between the lower and upper bounds of Bruno. It can be explained by the fact that the generalized Hashin-Shtrikman and the simplest upper and lower bounds are valid for all isotropic composites, whereas the bounds of Bruno incorporate details of the microstructure (see e.g. [16])

(ii) for the composite with inhomogeneity phase more conducting than matrix phase and with $\mathrm{HC}$ imperfect interfaces between them, the lower bound of Hashin-Shtrikman and

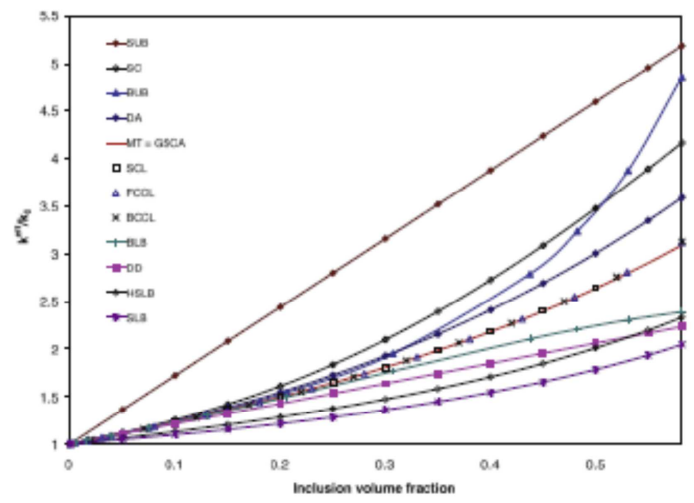

Fig. 4. Estimations and bounds of the effective thermal conductivity versus the inhomogeneity volume fraction of the two-phase composite with $\mathrm{HC}$ imperfect interfaces, $k_{i} / k_{0}=0.2$ and $k_{i}^{*} / k_{0}=4$

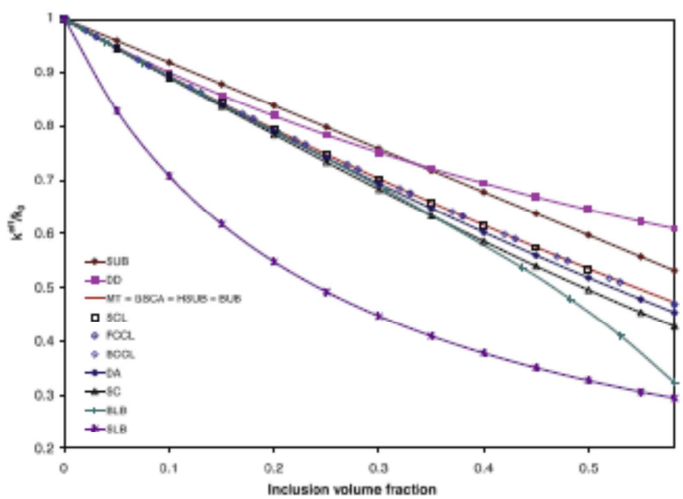

Fig 5. Estimations and bounds of the effective thermal conductivity of the twophase composite with LC imperfect interfaces, $\boldsymbol{k}_{i} / k_{0}=0.2$ and $\dot{\alpha}^{\infty} / h_{0}=0.125$.

the lower bound of Bruno for the effective thermal conductivity coincide with the estimations of the effective thermal conductivity derived by GSCA and MT schemes (Fig. 2). The similar observation can be made from Fig. 5 , for the case of the composite with inhomogeneity phase less conducting than matrix phase and with LC imperfect interfaces between matrix and inclusion phases, that the generalized HashinShtrikman and Bruno upper bounds for the effective thermal conductivity are identical to the results provided by GSCA and MT schemes.

(iii) Although the inhomogeneity phase is less conducting than the matrix phase, due to the effect of imperfect interfaces, the effective thermal conductivity in Fig. 4 increases when the inhomogeneity volume fraction augments. This is quite different from the relevant results of the classical case with perfect interfaces,

(iv) the effective thermal conductivities of periodic composites with simple, body-centered and face-centered cubic arrays derived by an exact method are very close to the ones estimated by MT or GSCA. The same observation has been men tioned in the recent work of Le Quang et al. [29] and Yvonnet et al. $[30]$ on the determination of the effective thermal conductivity of the periodic composites with $\mathrm{HC}$ imperfect interfaces by applying the method based on the fast Fourier transform (FFT) and the extended finite element method (XFEM). Consequently, we can conclude that the estimation using MT or GSCA is an excellent approximation for the effective thermal conductivity of the periodic composites with simple, body-centered and face-centered cubic arrays.

In the second example, to study the effects of the size of inhomogeneities on the effective thermal conductivity of mono-sphere composites, the inhomogeneity radius $R$ is set to vary while the inhomogeneity volume fraction is now kept constant with $c_{i}=0.1296$ or $c_{i}=0.4826$. The variation of the size of the inhomoge neities is characterized by a dimensionless parameter $\log _{2}\left[2 k_{s}^{(i)} /\left(k_{0} R\right)\right]=\log _{2}\left(2 \hat{k}_{s}^{(i)} / k_{0}\right)$ for HC imperfect interface or $\log _{2}\left[R /\left(k_{0} \alpha^{(i)}\right)\right]=\log _{2}\left(h_{0} / \hat{\alpha}^{(j)}\right)$ for LC imperfect interface. The thermal conductivities of the matrix and inhomogeneities are chosen to be such as $k_{i} / k_{0}=0.2$ for HC imperfect interface or $k_{i} / k_{0}=5$ for LC imperfect interface. Notice that these values of the phase properties have been used in the work of Lipton and Talbot [16]. In Figs. 6 and 7 are reported the estimations and bounds of the effective thermal conductivity of the two-phase composites with HC 


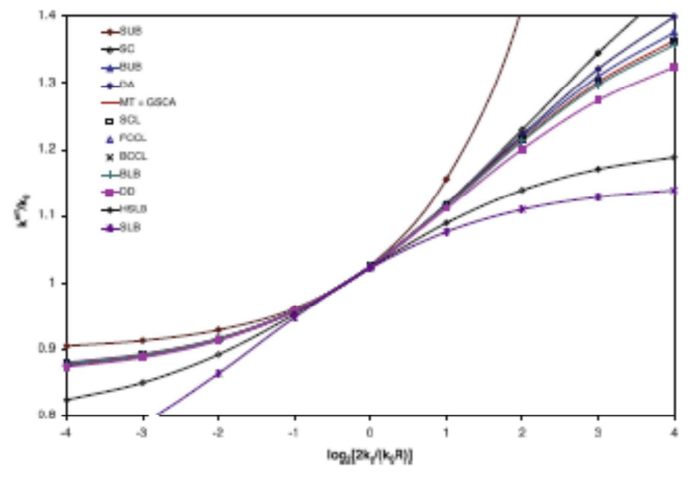

Fig. 6. Size-dependence for the estimations and bounds of the effective thermal conductivity of the two-phase composite with HC imperfect interfaces, $k_{i} / k_{0}-0.2$ and $c_{i}=0.1296$

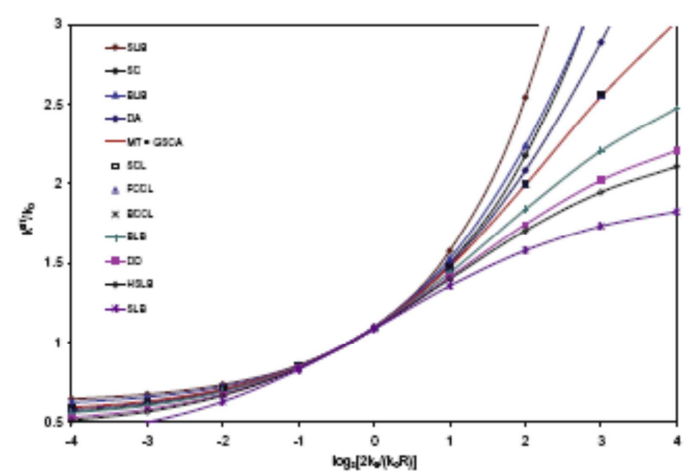

Fig. 7. Size-dependence for the estimations and bounds of the effective therm conductivity of the two-phase composite with HC imperfect interfaces, $k_{i} / k_{0}=0.2$ and $c_{i}=0.4826$

imperfect interfaces versus the parameter $\log _{2}\left[2 k_{s}^{(i)} /\left(k_{0} R\right)\right]=$ $\log _{2}\left(2 \hat{k}_{s}^{(i)} / k_{0}\right)$. Similarly. Figs. 8 and 9 illustrate the variation of the estimations and bounds of the effective thermal conductivity of the two-phase composites with LC imperfect interfaces in terms of $\log _{2}\left[R /\left(k_{0} \alpha^{(j)}\right)\right]=\log _{2}\left(h_{0} / \alpha^{(i)}\right)$. It can be seen from Figs. 6-9 that the estimations for the effective thermal conductivity derived by the micromechanical models described in Section 4, except the DD scheme, respect the simplest lower and upper bounds as well as the lower and upper generalized Hashin-Shtrikman bounds. However, as in the first example, owing to the fact that the Bruno lower and upper bounds involve more details about the microstructure, the estimations for the effective thermal conductivity are not always situated between them.

Next, in order to investigate the effective thermal conductivity of a multi-type-inclusion composite, we consider, in the third example, a composite consisting of a host matrix phase in which the spherical inhomogeneities of two different types, $k_{s}^{(1)}=2 \hat{k}_{s}^{(2)}$ (or $\hat{\alpha}^{(1)}=2 \dot{\alpha}^{(2)}$ ), are introduced. As in the first example, the conductivities of the inhomogeneity and matrix phases are chosen to be such as $k_{i} / k_{0}=5$ for the case where the inhomogeneity phase is more conducting than matrix phase and $k_{4} / k_{0}=0.2$ for the case where the inhomogeneity phase is less conducting than matrix

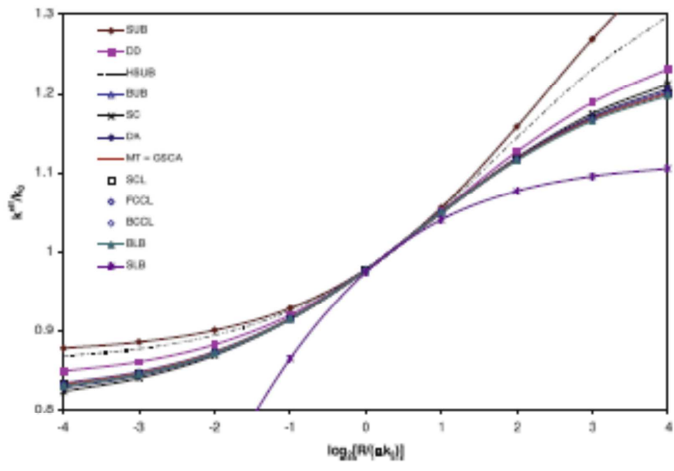

Fig. 8. Size-dependence for the estimations and bounds of the effective thermal cond uctivity of the two-phase composite with LC imperfect interfaces, $k_{1} / k_{0}=5$ and $c_{i}=0.1296$.

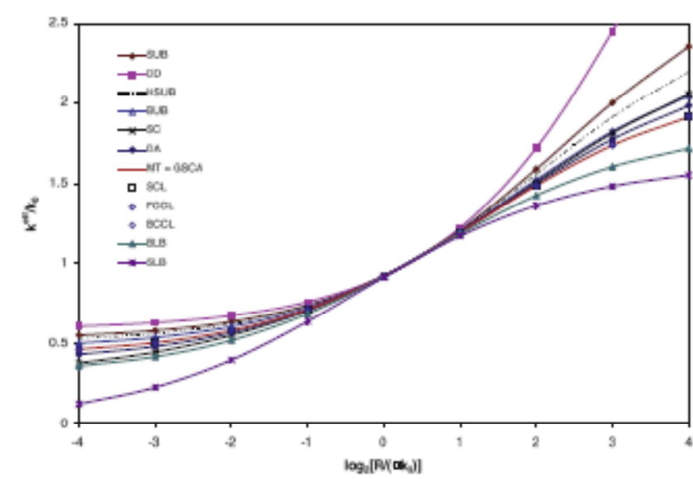

Fig. 9. Size-dependence for the estimations and bounds of the effective thermal conductivity of the two-phase composite with LC imperfect interfaces, $k_{0} / k_{0}-5$ and $c_{i}=0.4826$

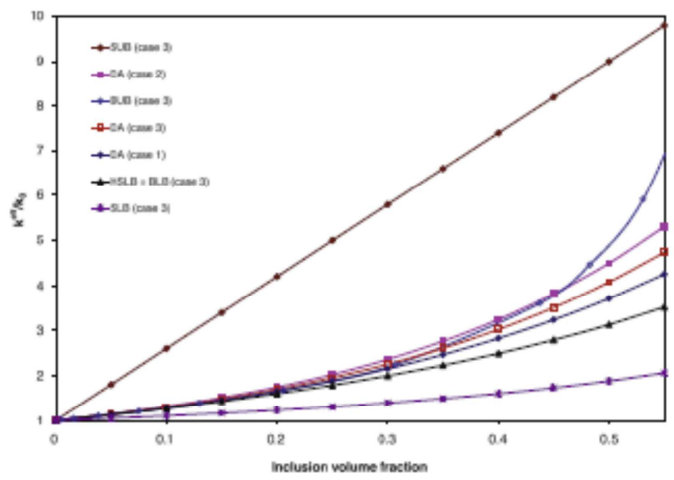

Fig. 10. Differential approximations and bounds of the effective thermal conductivity versus the inhomogeneity volume fraction of the composite with $\mathrm{HC}$ imperfect interfaces and $k_{j} / k_{0}-5, k_{k}^{3} / k_{0}=4$ (case 1); $k_{k} / k_{0}=5, k_{2}^{\prime} / k_{0}=8$ (case 2): $k_{2} / k_{0}=k_{2} / k_{0}=5, k_{3}^{(2)} / k_{0}=2 k_{1}^{(1)} / k_{0}-8$ and $c_{1}=c_{2}=\frac{1}{2} c_{i}$ (case 3 ). 


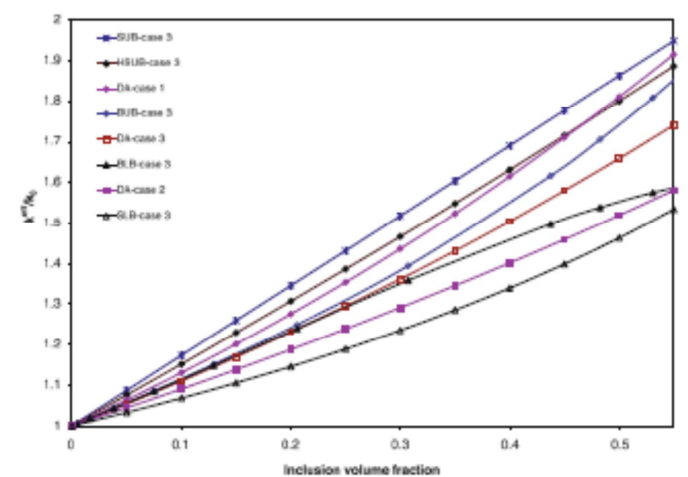

Fig. 11. Differential approximations and bounds of the effective thermal conductivity versus the in homogeneity volume fraction of the composite with LC imperfect interfaces and $k_{0} / k_{0}=5, \dot{\alpha}^{2} / h_{0}=0.125$ (case 1); $k_{4} / k_{0}=5, \dot{\alpha}^{\infty} / h_{0}=0.25$ (case 2); $k_{1}$ j $\left.k_{0}-k_{2} / k_{0}-5, \dot{\alpha}^{2}\right) / h_{0}=2 \dot{\alpha}^{(1)} / h_{0}=0.25$ and $c_{1}=c_{2}=\frac{1}{2} c_{1}$ (case 3 ).

phase. More precisely, by assuming that the inhomogeneity phase is more conducting than the matrix phase and by applying the differential approximation scheme, we plot in Figs. 10 and 11 the normalized effective thermal conductivity of the composite with two kinds of inhomogeneities, $\hat{k}_{s}^{(1)}=2 \hat{k}_{s}^{(2)}$ (or $\hat{\alpha}^{(1)}=2 \hat{\alpha}^{(2)}$ ), and two types of imperfect interfaces in terms of the total inhomogeneity volume fraction $c_{1}=2 c_{1}=2 c_{2}$ (case 3 ). These normalized effective thermal conductivities are compared first with the corresponding simplest lower and upper bounds (SLB and SUB), generalized $\mathrm{Ha}$ shin-Shtrikman lower and upper bounds (HSLB and HSUB) and Bruno lower and upper bounds (BLB and BUB) and then matched with the ones obtained from the composite containing spherical inclusions having $\hat{k}_{s}^{(2)}\left(\hat{\alpha}^{(2)}\right)$ (case 1 ) and that having $\hat{k}_{s}^{(1)}\left(\hat{\alpha}^{(1)}\right)$ (case 2 ). In a similar manner, the relevant results of the normalized effective thermal conductivities are depicted in Figs, 12 and 13 versus the total inhomogeneity volume fraction $c_{i}$ for the case where the inhomogeneity phase is less conducting than the matrix phase.

The fourth example consists of a composite made of a host iso tropic matrix phase of conductivity $k_{0}$ and resistivity $h_{0}=1 / k_{0}$, in which the spherical anisotropic inhomogeneities are embedded.

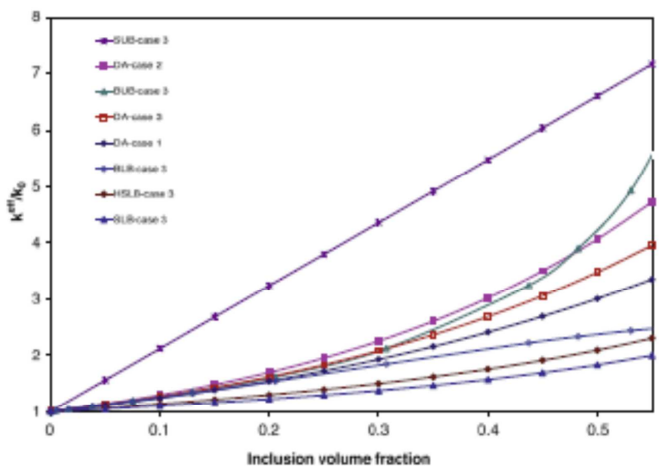

Fig. 12. Differential approximations and bounds of the effective thermal conductivity versus the inhomogeneity volume fraction of the composite with $\mathrm{HC}$ imperfect interfaces and $\boldsymbol{k}_{i} / \boldsymbol{k}_{0}=0.2, \hat{k}_{i}^{\phi} / \boldsymbol{k}_{0}=4$ (case 1); $\boldsymbol{k}_{i} / \boldsymbol{k}_{0}=0.2, \hat{k}^{9} / \mathbf{k}_{0}=8$ (case 2); $\left.k_{1} / k_{0}-k_{2} / k_{0}=0.2, k_{1}^{2}\right) / k_{0}=2 k_{3}^{(1)} / k_{0}=8$ and $c_{1}=c_{2}=\frac{1}{2} c_{i}$ (case 3).

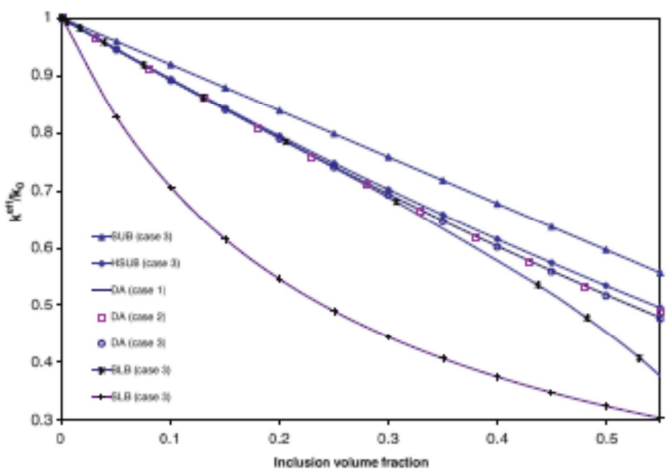

Fig 13. Differential approximations and bounds of the effective thermal conductivity versus the inhom ogeneity volume fraction of the composite with LC imperfect interfaces and $k / k_{0}=0.2, \hat{\alpha}^{\phi} / h_{0}=0.125$ (case 1 ); $k^{\prime} / k_{0}=0.2, \alpha^{\alpha} / h_{0}=0.25$ (case 2); $k_{1} / k_{0}=k_{2} / k_{0}=0.2, \dot{\alpha}^{(2)} / h_{0}=2 \dot{\alpha}^{(1)} / h_{0}=0.25$ and $c_{1}=c_{2}=\frac{1}{2} c_{i}$ (case 3).

Relative to an appropriate Cartesian coordinate basis, without loss of generality, the conductivity and resistivity of the inhomogeneity phase are assumed to be diagonal and denoted as $\mathbf{K}^{\left({ }^{(j)}\right.}=\operatorname{diag}\left[k_{1}^{(j)}, k_{2}^{(i)}, k_{3}^{(t)}\right]$ and $\mathbf{H}^{(t)}=\operatorname{diag}\left[h_{1}^{(i)}, h_{2}^{(i)}, h_{3}^{(t)}\right]$, respectively. The interfaces between the matrix and inhomogeneity phases are described either by the $\mathrm{HC}$ or LC imperfect interface model. In order to account for the anisotropy due to the spherical inhomogeneities on the effective thermal conductivity of the composite, we introduce an orientation distribution function (ODF), denoted as $W(\theta, \varphi)$, which satisfies the following integration condition

$\frac{1}{4 \pi} \int_{\varphi=0}^{2 \pi} \int_{\theta-0}^{\pi} W(\theta, \varphi) \sin \theta d \theta d \varphi=1$.

Moreover, in the present example of transversely isotropic orientation distributions around the axe $x_{3}$, the ODF is expressed as $W(\theta)$. More precisely, we assume that $W(\theta)$ takes the form (see, e.g., $[31,32]$ etc.)

$W(\theta)=\frac{\sigma \cosh (\sigma \cos \theta)}{\sinh (\sigma)}$,

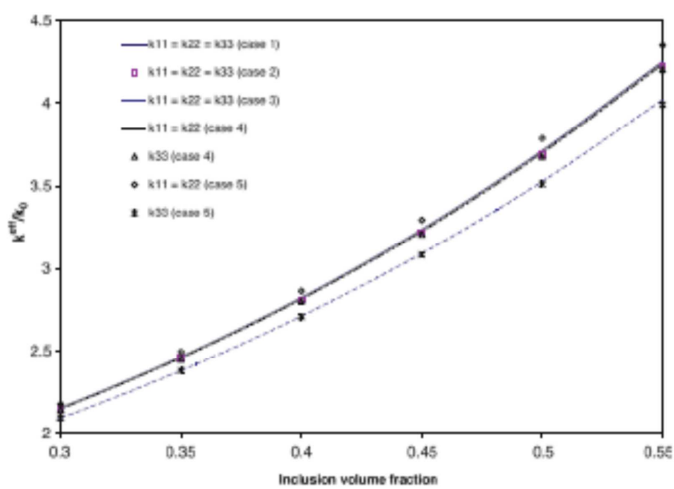

Fig 14. Differential approximations for the effective thermal conductivity versus the inhomogeneity volume fraction of the composite with HC imperfect interfaces

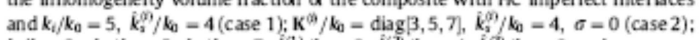

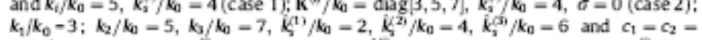

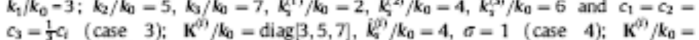
diag $3,5,7), k_{i}^{\infty} / k_{0}=4, \sigma=10$ (case 5) 


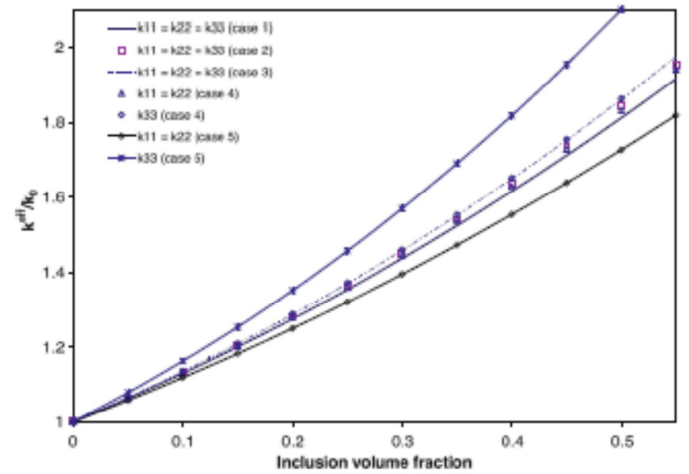

Fig. 15. Differential approximations for the effective thermal conductivity versus the inhomogeneity volume fraction of the composite with LC imperfect interfac

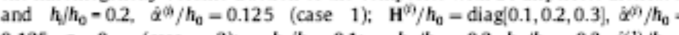
$0.125, \sigma=0 \quad$ (case 2); $\quad h_{1} / h_{0}=0.1 ; \quad h_{2} / h_{0}=0.2, h_{3} / h_{0}=0.3, \alpha^{11} / h_{0}$ $0.1, \alpha^{(2)} / h_{0}=0.125, \alpha^{(1)} / h_{0}=0.15$ and $c_{1}=c_{2}=c_{3}=\frac{1}{3} c_{i}$ (case 3); $\mathbf{H}^{n} / h_{0}$ diag $[0.1,0.2,0.3], \dot{\alpha}^{\infty} / h_{0}=0.125, \sigma=1 \quad$ (case 4 ); $\quad \mathbf{H}^{0} / h_{0}=\operatorname{diag}[0.1,0.2,0.3]$
$\dot{\alpha}^{\infty} / h_{0}=0.125, \sigma=10$ (case 5).

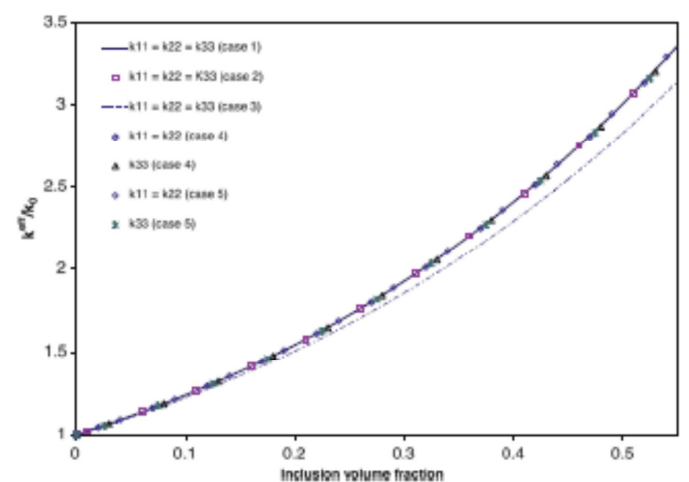

Fig. 16. Differential approximations for the effective thermal conductivity versus the inhomogeneity volume fraction of the composite with $\mathrm{HC}$ imperfect interfaces and $k_{i} / k_{0}=0.2, k^{6} / k_{0}=4$ (case 1$) ; K^{3} / k_{0}=\operatorname{diag}[0.1,0.2,0.3), k^{6} / k_{0}=4, \sigma=0$

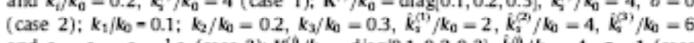

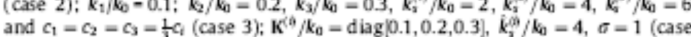
4): $\boldsymbol{\kappa}^{(\alpha)} / \mathbf{k}_{0}=\operatorname{diag}[0.1,0.2,0.3), \tilde{k}_{2}^{(n)} / \mathbf{k}_{0}=4, \sigma=10$ (case 5)

where $\sigma$ is a scalar parameter. In particular, when $\sigma=0$, it is imme diate from (78) that $W(\theta)=1$, or equivalently, the anisotropic inhomogeneities are identically distributed in all directions. With the foregoing expression of $W(\theta)$, in Figs. 14-17, the normalized effective principal thermal conductivities, $k_{11} / k_{0}, k_{22} / k_{0}$ and $k_{33} / k_{0}$ are plotted in terms of the inhomogeneity volume fraction $c_{i}$ with $\sigma=0$ (case 2), $\sigma=1$ (case 4) and $\sigma=10$ (case 5) for the two cases where the inhomogeneity phase is either more or less conducting than matrix phase and with HC or LC imperfect interfaces model. Then, these estimations for the effective thermal conductivity are compared with the one provided by the composite made of the same isotropic matrix phase in which the mono-sized spherical and isotropic inhomogeneities with conductivity $k^{(i)}=\frac{1}{3}\left(k_{1}^{(i)}+k_{2}^{(j)}+k_{3}^{(j)}\right)$ and resistivity $h^{(j)}=\frac{1}{3}\left(h_{1}^{(i)}+h_{2}^{(i)}+h_{3}^{(i)}\right)$ are inserted (case 1). Finally, for comparison, we show also in Figs. 14-17 the effective thermal conductivity of the composite

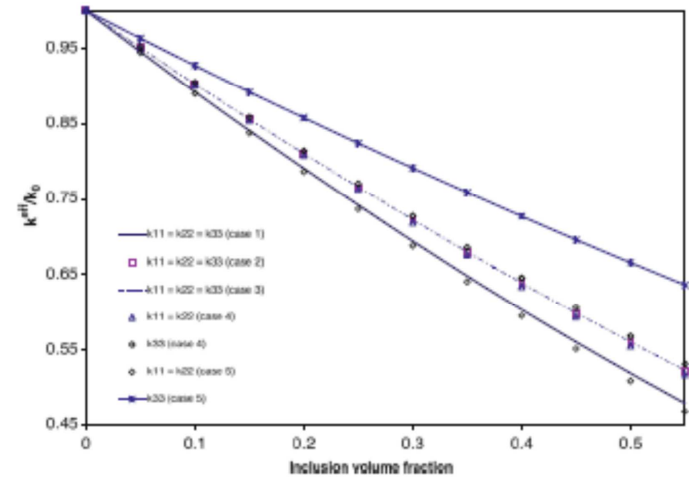

Fig. 17. Differential approximations for the effective thermal conductivity versus the inhomogeneity volume fraction of the composite with LC imperfect interfaces and $h_{i} / h_{0}=5, \alpha^{6} / h_{0}=0.125$ (case 1); $\left.\mathbf{H}^{\boldsymbol{W}} / h_{0}=\operatorname{diag}[2,5,8], \dot{\alpha}^{2}\right) / h_{0}=$ $0.125, \sigma=0$ (case 2); $h_{1} / h_{0}-2 ; h_{2} / h_{0}=5, h_{0} / h_{0}=8, \alpha^{1} / h_{0}=0.1, \alpha^{2} / h_{0}=$ $0.125, \dot{\alpha}^{(3)} / h_{0}=0.15$ and $c_{1}=c_{2}=c_{3}=\frac{1}{3} c_{i}$ (case 3): $\mathbf{H}^{\prime \prime} / h_{0}=\operatorname{diag}[2,5,8$, $\dot{\alpha}^{\infty} / h_{0}=0.125, \sigma=1$ (case 4): $\mathbf{H}^{\infty} / h_{0}=\operatorname{diag}[2,5,8], \alpha^{\infty} / h_{0}=0.125, \sigma=10$ (case

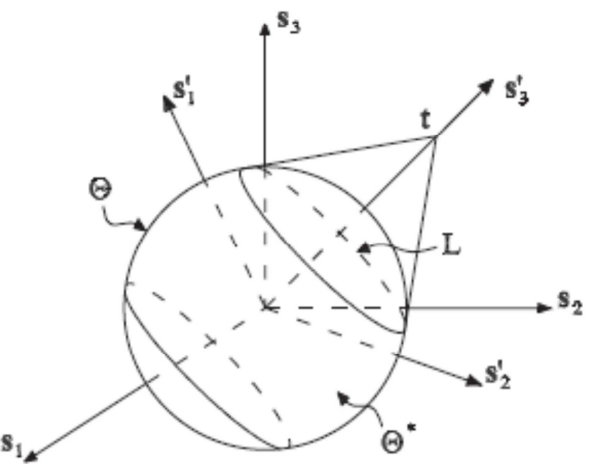

Fig. 18. The subdomains $\Theta^{*}$ and $L$ of $\Theta$ satisfying the condition $|\mathbf{s} \cdot \mathbf{t}| \zeta 1$ and s. $\mathbf{t}=1$ and the chosen new coord inate system $\left\{s_{1}, s_{2}, s_{3}\right\}$ for a given vector $\mathbf{t}$.

containing three isotropic inhomogeneity phases with $c_{1}=c_{2}=$ $c_{3}=c_{l} / 3, k^{(1)}=k_{1}^{(i)}, k^{(2)}=k_{2}^{(j)}, k^{(3)}=k_{3}^{(i)}, h^{(1)}=h_{1}^{(i)}, h^{(2)}=h_{2}^{(j)} \quad$ and $h^{(3)}=h_{3}^{(i)}$ (case 3 ). Note that in this case, the three spherical inhomogeneity phases are chosen to be such as $\frac{1}{3}\left(\hat{k}_{s}^{(1)}+\hat{k}_{s}^{(2)}+\hat{k}_{s}^{(3)}\right)=\hat{k}_{s}^{(3)}$ or $\frac{1}{3}\left(\hat{\alpha}^{(1)}+\hat{\alpha}^{(2)}+\hat{\alpha}^{(3)}\right)=\hat{\alpha}^{(i)}$.

\section{Concluding remarks}

The closed-form expressions for the effective conductivity or resistivity tensor of anisotropic multiphase composites with imperfect interfaces have been derived by using the Eshelby's formalism and dilute solution results. Unlike the results derived previously for the effective properties of the inhomogeneous materials with imperfect interfaces, the results given in the present paper for thermal conduction hold for any thermal anisotropy of materials constituting the matrix and the inhomogeneities. Moreover, the results show that, as in the classical case with perfect interfaces, the effective conductivity estimated by the differential 
scheme for multiphase multi-type-inclusion never violates the generalized Hashin-Shtrikman bounds. In view of the conclusion (iv) made in Section 5, the estimation obtained by the MT or GSCA scheme can be viewed an excellent approximation for the effective thermal conductivity of the two-phase periodic composites with simple, body-centered and face-centered cubic arrays.

Finally, due to the physical analogy existing between different transport phenomena, the results obtained by the present work for the thermal conduction phenomenon are straightforwardly transposable, for example, to electric conduction, dielectrics, magnetism, diffusion and flow in porous media. By using the close relation existing between the depolarization tensor in electrostatics and Eshelbys conduction tensor under consideration (see, eg., [33,34]), all results derived in this work for the thermal conduction phenomenon can be directly generalized to the electromagnetic phenomenon. Additionally, since a mathematical correspondence between anti-plane elasticity and 2D thermal conduction exists, new results for anisotropic anti-plane elasticity can be deduced directly from the $2 \mathrm{D}$ anisotropic results derived above.

\section{Acknowledgements}

We thank the University Paris-Est and Vietnam's Nafosted for providing support for D.C. Pham.

\section{Appendix A}

This appendix aims at showing the explicit computation of the components $D_{m}(\mathbf{x})$ and $N_{\text {ip }}(\mathbf{x})$ defined as Eqs. (27) and (28). For clarity, for their derivation and the relevant technical details, the reader can refer to [11]. The main results are presented below

(i) Case when $\omega^{(i)}$ is a spherical inhomogeneity with radius $R_{i}$

For interior points of the inclusion $\omega^{(i)}$, the expressions of $D_{m y}(\mathbf{x})$ and $N_{\text {nip }}(\mathbf{x})$ are given by

$D_{m j}(\mathbf{x})=\frac{1}{4 \pi} \int_{-1}^{1} d s_{3} \int_{0}^{2 \pi} g_{m j}(\mathbf{s}) d \zeta$,

$N_{\text {mip }}(\mathbf{x})=\frac{3 R_{i}}{4 \pi} \int_{-1}^{1} d s_{3} \int_{0}^{2 \pi} s_{p}(\mathbf{s} \cdot \mathbf{t}) g_{n j}(\mathbf{s}) d \zeta$

where $s$ is a unit vector whose components are expressed as

$s_{1}=\left(1-s_{3}^{2}\right)^{1 / 2} \cos \zeta, \quad s_{2}=\left(1-s_{3}^{2}\right)^{1 / 2} \sin \zeta$

and $\mathbf{t}$ and $g_{m y}(\mathbf{s})$ are, hereafter, defined as

$\mathbf{t}=\frac{\mathbf{x}}{R_{i}}, \quad g_{m j}(\mathbf{s})=\frac{s_{m} s_{j}}{K_{n q}^{0} s_{n} s_{p}}$.

For exterior points of the inclusion $\omega^{(i)}$, by introducing a new coordinate system $\left\{s_{1}^{\prime}, s_{2}^{\prime}, s_{3}^{\prime}\right\}$ in which $s_{3}^{\prime}$-axis is taken in the direction of $t$, and $s_{1}^{\prime}$-and $s_{2}^{\prime}$-axis are given along two perpendicular directions in the plane normal to $\mathbf{t}$ (see Fig. 18), we can then express the relation between $\left\{s_{1}, s_{2}, s_{3}\right\}$ and $\left\{s_{1}, s_{2}, s_{3}\right\}$ by

$s_{i}=\Xi_{i j} s_{j}$,

where $\Xi_{i j}$ are the direction cosines between the $s_{i}$ - and $s_{j}$-axis. Since

$g_{p j}(\mathbf{s})=g_{p j}\left(\Xi_{1 m} s_{m}^{\prime}, \Xi_{2 m} s_{m}^{\prime}, \Xi_{3 m} s_{m}^{\prime}\right)=g_{p j}^{\prime}\left(\mathbf{s}^{\prime}\right)$

and by setting

$s_{1}^{\prime}=\left(1-s_{3}^{2}\right)^{1 / 2} \cos \zeta^{\prime}, \quad s_{2}^{\prime}=\left(1-s_{3}^{2}\right)^{1 / 2} \sin \zeta^{\prime}$,

the expressions of $D_{m}(\mathbf{x})$ and $N_{n j p}(\mathbf{x})$ take the following form $\begin{aligned} D_{m j}(\mathbf{x})= & \frac{1}{4 \pi} \int_{-1 / t \mid t}^{1 / t \mid} d s_{3}^{\prime} \int_{0}^{2 \pi} g_{m j}^{\prime}\left(\mathbf{s}^{\prime}\right) d \zeta^{\prime} \\ & -\frac{1}{2 \pi} \int_{0}^{2 \pi}|\mathbf{t}|^{-1}\left\{g_{m j}^{\prime}\left(s^{\prime}\right)\right\}_{\xi_{3}-1 / t \mid t} d \zeta^{\prime},\end{aligned}$

$N_{\text {mip }}(\mathbf{x})=\frac{3 R_{i}}{4 \pi} \Xi_{p \gamma} \int_{-1 / t \mid t}^{1 / t \mid} d s_{3}^{\prime} \int_{0}^{2 \pi} s_{\gamma}^{\prime} s_{3}^{\prime}|t| g_{n j}^{\prime}\left(s^{\prime}\right) d \zeta^{\prime}$

$-\frac{R_{i}}{2 \pi} \Xi_{p y} \int_{0}^{2 \pi} \mid \mathbf{t}^{-1}\left\{s_{\gamma}^{\prime} g_{\pi j}^{\prime}\left(s^{\prime}\right)\right\}_{s_{3}-1 / t} d \zeta^{\prime}$.

(ii) Case when $\omega^{(i)}$ is a circular inhomogeneity with radius $R_{\text {fi }}$.

For interior points of the inclusion $\omega^{(i)}, D_{m j}(\mathbf{x})$ and $N_{n j p}(\mathbf{x})$ are determined by

$D_{m j}(\mathbf{x})=\frac{1}{2 \pi} \int_{0}^{2 \pi} g_{m j}\left(s_{1} / a_{1}, s_{2} / a_{2}, 0\right) d \zeta$,

$N_{\text {mip }}(\mathbf{x})=\frac{R_{i}}{\pi} \int_{0}^{2 \pi} s_{p}\left(s_{1} t_{1}+s_{2} t_{2}\right) g_{\text {mij }}\left(s_{1} / a_{1}, s_{2} / a_{2}, 0\right) d \zeta$,

with

$s_{1}=\cos \zeta, \quad s_{2}=\sin \zeta$.

For exterior points of the inclusion $\omega^{(i)}, D_{m}(\mathbf{x})$ and $N_{\text {mip }}(\mathbf{x})$ are calculated by

$\begin{aligned} D_{m j}(\mathbf{x})= & \frac{1}{4 \pi} \int_{-1 / t \mid}^{1 / t \mid} d s_{3}^{\prime} \times \int_{0}^{2 \pi} g_{m j}\left(\Xi_{1 \gamma} s_{\gamma}^{\prime}, \Xi_{2 \gamma} s_{\gamma}, 0\right) d \zeta^{\prime} \\ & -\frac{1}{2 \pi} \int_{0}^{2 \pi}|\mathbf{t}|^{-1}\left\{g_{m j}\left(\Xi_{1 \gamma} s_{\gamma}, \Xi_{2 \gamma} s_{\gamma}^{\prime}, 0\right)\right\}_{\zeta_{\zeta}-1 /\left.t\right|^{\prime}} d \zeta^{\prime},\end{aligned}$

$N_{n j p}(\mathbf{x})=\frac{3}{4 \pi} \Xi_{p} R_{l} \int_{-1 / t \mid}^{1 / 1 t} d s_{3}^{\prime} \int_{0}^{2 \pi} s_{\gamma}^{\prime} s_{3}^{\prime}|t| g_{w j}\left(\Xi_{1 m} s_{m}^{\prime}, \Xi_{2 m} s_{m}^{\prime}, 0\right) d \zeta^{\prime}$

$-\frac{1}{2 \pi} \Xi_{N i} R_{l} \int_{0}^{2 \pi}|\mathrm{t}|^{-1}\left\{s_{\gamma} g_{m i}\left(\Xi_{1 m} s_{m}^{\prime}, \Xi_{2 m} s_{m}^{\prime}, 0\right)\right\}_{s_{3}=1 / \pi|t|} d \zeta^{\prime}$,

with

$s_{1}^{\prime}=\cos \zeta^{\prime}, \quad s_{2}=\sin \zeta^{\prime}$.

\section{References}

[1] P.L Kapitza, The study of heat transfer in helium II. J. Phys. (USSR) 4 (1941)

181-210.
[2] E. Sanchez-Palencia, Comportement limite drun problème de transmission à travers une plaque faiblement conductrice, C. R Acad. Sci. Paris Ser. A27

3] H. Pham Huy, E. Sanchez-Palencia Phénomènes de transmission à travers des couches minces de conductivité elevée J. Math. Anal. Appl. 47 (1974) 284-309 P. Bavik, On the modelling of thin interface layers in elastic and acoustic Y.

[5] Y. Benveniste, T. Miloh. The effective conductivity of composites with imperfect thermal contact at constituent interfaces, Int. J. Eng. Sci 24 (1986)

6] T. Miloh Y

$Y$ Benveniste On the effective conductivity of composites with ellipsoidal inhomogeneities and highly conducting interfaces, Proc. $R$ Soc. Lond. 455 (1999) 2687-2706.

[7] Z Hashin. Thin interphase/imperfect interface in conduction, I Appl. Phys. 84 (2001) 2261-2267.

8] Y. Benveniste, A general interface model for a three-dimensional curved thin anisotropic interphase between two anisotropic media.J. Mech. Phys Solids 54 (2006) 708-734.

(9) S-T. Gu, Q-C He, Interfacial discontinuity relations for coupled multifield phenomena and their application to the modelling of thin interphases as

imperfect interfaces, J. Mech. Phys. Solids 59 (2011) 1413-1426.
[10] H. Le Quang. G. Bonnet, Q-C. He, Size-dependent Eshelby tensor fields and effective conductivity of composites made of anisotropic phases with highly conducting imperfect interfaces, Phys. Rev. B 81 (2010) 064203. 
[11] H. Le Quang. Q-C He, G. Bonnet, Eshelby's tensor fields and effective conductivity of composites made of anisotropic phases with Kapitza's interface thermal resistance, Philos. Mag. 91 (2011) 3358-3392

[12] R Lipton, B. Vernescu, Composites with imperfect interface, Proc. Phys. Soc. 6) 329-35

[13] R Lipton, B. Vernescu, Critical radius, size effects and inverse problems for composites with imperfect interface, J. Appl Phys. 79 (1996) 8964-8966.

[14] R Lipton, Reciprocal, relations bounds, and size effects for composites with highly conducting interface, SAMM J. Appl. Math 57 (1997) 347-363

$R$ Lipton Variational methods, highly conducting interface. J. Mech. Phys. Solids 45 (1997) 361-384.

[16] R. Lipton. DRS. Talbot, Bounds for the effective conductivity of a composite

[17] S. Torquato, M. D. Rintoul, Effect of the interface on the properties of composite media, Phys. Rev. Lett. 75 (1995) $4067-4070$

18] H. Cheng. S. Torquato, Effective conductivity of dispersion of spheres with a superconducting interface. Proc Roy. Soc. London A453 (1997) 1331-1344

9] R Hill, Discontinuity relations in mechanics of solids, in: IN. Sneddon, R. Hill (Eds.), Progress in Solid Mechanics, vol. 2, North-Holland, Amsterdam. 1999,

[20] D.P. 247 . Hasselman. LF. Johnson, fffective thermal conductivity of composite with interfacial thermal barrier resistance. J. Compos. Mater. 21 (1987) 508515.

21] H. Cheng. S. Torquato, Effective conductivity of periodic arrays of spheres with interfacial resistance, Proc. Roy. Soc London A53 (1997) 145-161.

[22] CW. Nan. R Brringer, D.R. Clarke, H. Gleiter, fffective thermal conductivity of particulate composites with interfacial thermal resistance, J. Appl. Phys. 81 (1997) $6692-6699$ [23] J.R Dryden, Elastic constants of spherulitic polymer, J. Mech. Phys. Solids 36
[24] T. Chen, Thermoelastic properties and conductivity of composites reinforced by spherical partides, Mech. Mater. 14 (1993) 257-268.

[25] A.N. Norris AJ Callegari, P.J Sheng, Generalized differential effective medium theory. J. Mech. Phys. Solids 33 (1985) 525-543.

Pham, Differential multiphase models for polydispersed spheroidal inclusions: thermal conductivity and effective viscosity. Int. J. Eng. Sci. 38 (2000) 73-88.

[27] D.C. Pham. Weighted effective medium approximations for conductivity of random com posites, International purnal of Heat and Maes Tran sfer 51 (2008)

[28] O.P. Bruna. The effective conductivity of strongly heterogeneous composites, Proc. Roy. Soc London A433 (1991) 353-381. 9] H. Le Quang T.-L. Phan, G. Bonnet, Effective thermal conductivity of periodic

30] I. Yvonnet, Q-C. He, C Toulemonde, Numerical modelling of the effective conductivities of composites with arbitrarily shaped inclusions and highly

31] CM. Sayers. The elastic anisotropy of shales. J. Geop 28ys. Res 99 (1994) 767774 .

[32] TA. Johansen, M. Jakobsen, B.O. Ruud, Estimation of internal structure and anisotropy of shales from borehole data. Tech. rep., SRC Report No. 01-003,

[33] WS. Weiglhoder, A. Lakhtakia, B. Michel, Maxwell Garnett and Bruggeman formalisms for a particulate composite with bianisotropic host medium.

[34] S. Giordano, P. Palla. Dielectric behavior of anisotropic inhomogeneities: interior and exterior point Eshelby tensors, J. Phys. A : Math. Theor. 41 (2008) 